\title{
RADAR: Self-Configuring and Self-Healing in Resource Management for Enhancing Quality of Cloud Services
}

\author{
Sukhpal Singh Gill ${ }^{1 *}$, Inderveer Chana ${ }^{2}$ and Maninder Singh ${ }^{3}$ and Rajkumar Buyya ${ }^{4}$ \\ ${ }^{1,4}$ Cloud Computing and Distributed Systems (CLOUDS) Laboratory \\ School of Computing and Information Systems \\ The University of Melbourne, Australia \\ ${ }^{2,3}$ Computer Science and Engineering Department, \\ Thapar Institute of Engineering and Technology, Patiala, Punjab, India \\ ${ }^{1}$ sukhpal.gillaunimelb.edu.au, 2 inderveerathapar.edu, 3msingh@thapar.edu, \\ 4rbuyyadunimelb.edu.au \\ ${ }^{*}$ Corresponding Author
}

\begin{abstract}
Cloud computing utilizes heterogeneous resources that are located in various datacenters to provide an efficient performance on pay per use basis. However, existing mechanisms, frameworks and techniques for management of resources are inadequate to manage these applications, environments and the behavior of resources. There is a requirement of Quality of Service (QoS) based autonomic resource management technique to execute workloads and deliver cost-efficient and reliable cloud services automatically. In this paper, we present an intelligent and autonomic resource management technique named RADAR. RADAR focuses on two properties of self-management, firstly self-healing that handles unexpected failures and secondly self-configuration of resources and applications. The performance of RADAR is evaluated in the cloud simulation environment and the experimental results show that RADAR delivering better outcomes in terms of execution cost, resource contention, execution time, SLA violation while delivers reliable services.
\end{abstract}

Keywords: Cloud Computing, Resource Provisioning, Self-management, Self-healing, Self-configuring, Quality of Service, Resource Scheduling, Service Level Agreement

\section{Introduction}

Cloud computing offers various services like Software as a Service (SaaS), Platform as a Service (PaaS), and Infrastructure as a Service (IaaS). However, providing dedicated cloud services that ensure various Quality of Service (QoS) requirements of cloud user and avoid Service Level Agreement (SLA) violations is a difficult task. Based on the availability of cloud resources, dynamic services are provided without ensuring the required QoS [1]. To fulfill the QoS requirements of user applications, the cloud provider should change its ecosystem [2]. Self-management of cloud services is needed to provide required services and fulfill the QoS requirements of the user automatically.

Autonomic management of resources manages the cloud service automatically as per the requirement of the environment, therefore maximizing resource utilization and cost-effectiveness while ensuring the maximum reliability and availability of the service [3]. Based on human guidance, a self-managed system keeps itself stable in uncertain situations and adapts rapidly to new environmental situations such as network, hardware or software failures [4]. QoS based autonomic systems are inspired by biological systems, which can manage the challenges such as dynamism, uncertainty and heterogeneity. IBM's autonomic model [3] based cloud computing system considers MAPE-k loop (Monitor, Analyze, Plan and Execute) and its objective is to execute workloads within their budget and deadline by satisfying the QoS requirements of the cloud consumer. An autonomic system considers the following properties while managing cloud resources [1] [2] [3]:

- Self-healing recognizes, analyses and recovers from the unexpected failures automatically.

- Self-configuring adapts to the changes in the environment automatically.

In this paper, we have developed a technique for self-configuRing and self-heAling of clouD bAsed Resources, called $\boldsymbol{R A D A R}$, with the focus of two properties of autonomic-management that provide self-healing by handling unexpected failures and selfconfiguration of resources and applications. The performance of RADAR is evaluated in the cloud environment and the experimental results show that RADAR delivers better outcomes in terms of QoS parameters and delivers cost-efficient and reliable cloud services. The key contributions of this research work are outlined as follows:

i) RADAR provides self-configuration of resources and applications by the re-installation of outdated or missing components and self-healing is offered by managing unexpected faults or errors automatically.

ii) RADAR schedules the provisioned cloud resources automatically and optimizes user's QoS requirements, which improves the user satisfaction and reduces the human intervention. Therefore, the cloud providers provide effective cloud service delivery and avoid SLA violations.

iii) Based on self-managed properties of an autonomic system, RADAR offers algorithms for its four different phases (monitor, analyze, plan and execute). RADAR monitors QoS value continuously during workload execution, analyzes the alert in case of degradation of performance, plans an appropriate action to manage that alert and implements the plan to preserve the system's efficiency.

iv) RADAR reduces SLA violations, energy consumption and resource contention, and improves availability and reliability of cloud services when implemented in cloud environment. 
The rest of the paper is organized as follows. Section 2 presents the related work. Proposed technique is presented in Section 3. Sections 4 presents the performance evaluation and experimental results. Section 5 presents conclusions and future work.

\section{Related Work}

Autonomic resource management (also known as self-management) is a big challenge due to the discovery and allocation of a best workload-resource pair for execution of cloud workloads. As the literature on this topic is vast, we focus on self-configuring and self-healing of resources for enhancing the quality of cloud services during workload execution. Interested readers can find a detailed survey on the QoS-aware autonomic management of cloud resources in [1]. This section briefly discusses the related work of selfconfiguring and self-healing in the cloud environment.

\subsection{Self-Healing}

An early attempt to incorporate self-healing into cloud resource management is done by Chen et al. [4], they propose a self-healing framework (SHelp) for management of multiple application instances in a virtual cloud environment to reduce software failures. The authors apply error virtualization techniques and weighted rescue points to develop applications to avoid the faulty path. Further, SHelp uses a rescue point database, which stores the error handling information to decrease the forthcoming faults generated by similar bugs. SHelp improves the fault detection rate and recovers system quickly from faults, but it executes only homogeneous cloud workloads. Mosallanejad et al. [5] propose an SLA based Self-Healing (SH-SLA) model to develop hierarchical SLA for the cloud environment, which effectively monitors SLA and detects SLA violation automatically. Further, related SLAs (with same QoS requirements) communicate with each other in a hierarchical manner. SH-SLA model performs effectively in fault detection, but it is not able to prevent the fault occurrence, which reduces the user satisfaction. Similar work has been done by Mosallanejad et al. [12], who applies replication technique for fault management.

Alhosban et al. [6] propose Self-Healing Framework (SHF), which uses the previous history to detect the occurrence of faults in cloud-based systems. Moreover, SHF develops a recovery plan to avoid future faults generated by similar bugs, but it needs autonomic fault prevention mechanism to improve the performance of the system. Silva et al. [7] propose a Self-Healing Process (SHP) for effective management of operational workflow incidents on distributed computing infrastructures. Further, incident degrees of workflow activities (i.e. task failure rate due to application errors) are measured using different metrics such as data transfer rate, application efficiency and long-tail effect to detect faults occurring during execution of workloads. Moreover, Virtual Imaging Platform [8] is used to evaluate the performance of SHP, which demonstrates the improvement in execution time of workloads.

Li at al. [9] propose a Self-Healing Monitoring and Recovery (SHMR) conceptual model, which composes cloud services into valueadded services to fulfill the changing requirements of cloud users. SHMR works in three different steps: 1) monitor the working of the system to identify the occurrence of faults, 2) find out the properties of faults and 3) recover the fault using an undo strategy. Magalhaes and Silva [10] propose a Self-healing Framework for Web-based Applications (SFWA) to fulfill the user SLA and improve the resource utilization simultaneously through self-adaption of cloud infrastructure. Experimental results show that SFWA adjusts infrastructure dynamically to detect anomalies, which reduces the delay during workload execution. Similar work has been done by Xin et al. [11], who suggest that the combination of data analytics and machine learning can be utilized to improve automatic failure prediction for cloud-based environments.

Rios et al. [13] propose Application Modelling and Execution Language (AMEL) based conceptual model for self-healing, which models the multi-cloud applications in the distributed environment. Further, AMEL uses a security modeling language to design SLA in terms of security and privacy aspects. Azaiez and Chainbi [14] propose a Multi-Agent System Architecture (MASA) for self-healing of cloud resources by analyzing the resource utilization continuously. Further, a checkpointing strategy is used in MASA to manage the occurrence of faults and it only considers static checkpoint intervals for fault tolerance mechanism. The main difference between these works and ours is that none of them consider autonomic fault prevention mechanism for self-healing with dynamic checkpoint intervals, which is presented in this paper. The existing self-healing frameworks [4-12] focus on monitoring of faults to maximize fault detection rate, whereas our work focuses on fault detection as well as prevention mechanisms.

\subsection{Self-Configuring}

Sa et al. [15] examine the problem of fault tolerance for distributed systems, which deals with the accuracy and speed of detection of faults. The authors propose a QoS-based Self-Configuring (QoS-SC) framework, which uses feedback control theory for detection of faults automatically. MATLAB based performance evaluation testbed is used to validate the proposed framework. Maurer et al. [16] investigate the impact of SLA violation on resource utilization in autonomic cloud computing systems. Further, an Adaptive Resource Configuration (ARC) framework is proposed for the effective management of cloud resources to execute the synthetically generated workloads. As a part of their work, a case-based reasoning approach is used to maintain the execution details of the workload in a centralized database. The ARC framework improves the utilization of cloud resources. Their framework considers multiple resources such as bandwidth, storage, memory and CPU, while our model contains bundles of resources, i.e. VM instances. Salaun et al. [17] propose a Self-Configuration Protocol (SCP) for management of distributed applications in a cloud environment without using centralized database [16] and SCP measures the execution time of workloads with the different number of virtual machines. Similar work has been done by Etchevers et al. [18], who uses reconfigurable component-based systems to design distributed applications to minimize execution time, but it increases their configuration interdependencies. Panica et al. [19] and Wolinsky et al. [21] examine the problem of execution of legacy distributed applications using self-configuration of cloud infrastructure to maximize the availability and reliability of cloud services. 
Sabatucci et al. [20] investigate the concept of composing mashups of applications in the autonomic cloud environment. Sabatucci et al. [24] propose a Goal-Oriented Approach for Self-Configuring (GOASC) for automatic composing mashups of applications distributed over the geographical cloud environment. In GOASC, available functionalities are defined in terms of capabilities (for example: maximum reliability and availability) at the cloud user side, whereas mashup logic (maximum resource utilization and energy efficiency) is defined in terms of goals at the cloud provider side. Cordeschi et al. [21] propose an Energy-Aware SelfConfiguring (EASC) approach for virtualized networked data centers to execute workloads with the minimum value of energy consumption. As a part of their work, an energy-scheduler is also developed to enable scalable and distributed cloud environment for scheduling of energy-efficient cloud resources. Their approach considers homogenous workloads, while our model considers both homogenous and heterogeneous workloads.

Lama and Zhou [22] propose an Autonomic Resource allOcation MechAnism (AROMA) for effective management of cloud resources for workload execution, while satisfying their QoS requirements as described in SLA. Further, auto-configuration of Hadoop jobs and provisioning of cloud resources is performed using support vector machine-based performance model. AROMA uses previous details of resource execution for allocation of resources to new workloads, which improves utilization of resources. Konstantinou et al. [23] propose a Cost-aware Self-Configured (COCCUS) framework for effective management of cloud query services, which execute queries for optimization of cloud services in terms of QoS parameters. Initially, cloud user specifies his QoS requirements (budget and deadline constraint) and then COCCUS executes the user queries on the available set of resources within their deadline and budget. The details of every user and their query is maintained in a centralized component is called CloudDBMS.

Bu et al. [25] propose a VM based Self-Configuration (CoTuner) framework to provide a coordination between virtual resources and user applications using a model-free hybrid reinforcement learning approach. CoTuner handles the changing requirements of user workloads using knowledge guided exploration policy, which uses the information of memory and CPU utilization for selfmanagement of cloud resources to improve the learning process. Their framework considers scheduling of single queued workload, while our model considers clustering of workloads based on their QoS requirements. There is a large body of research devoted to self-configuration of cloud resources for execution of homogenous cloud workloads only [15-25]. However, the limited investigation has been done on the execution of heterogeneous workloads in the context of autonomic cloud computing. Further, there is a need to execute workloads without the violation of SLA. QoS-aware autonomic resource management approach (CHOPPER) [29] is an extended version of RADAR, which considers the self-optimization for improving QoS parameters and selfprotection against cyber-attacks as well.

\subsection{Comparison of RADAR with Existing Techniques}

The proposed technique (RADAR) has been compared with existing resource management techniques as described in Table 1. The existing research works have considered either self-healing or self-configuring, but none of the existing works consider self-healing and self-configuring simultaneously in a single resource management technique to the best of the knowledge of authors. Moreover, most of the existing work considers homogeneous cloud workloads. None of the existing work considers provisioning-based resource scheduling. Existing techniques consider the execution of single queued workload instead of clustering of workloads. RADAR schedules the provisioned resources for the execution of clustered heterogeneous workloads with maximum optimization of QoS parameters.

Table 1: Comparison of RADAR with Existing Resource Management Techniques

\begin{tabular}{|c|c|c|c|c|c|c|c|c|}
\hline \multirow[b]{2}{*}{ Technique } & \multicolumn{2}{|c|}{ Self-Healing } & \multicolumn{2}{|c|}{ Self-Configuring } & \multirow{2}{*}{$\begin{array}{l}\text { Heterogeneous } \\
\text { Workloads }\end{array}$} & \multirow{2}{*}{$\begin{array}{c}\text { Clustering } \\
\text { of } \\
\text { Workloads }\end{array}$} & \multirow{2}{*}{$\begin{array}{c}\text { Provisioning } \\
\text { based } \\
\text { Scheduling }\end{array}$} & \multirow[b]{2}{*}{ QoS Parameters } \\
\hline & $\begin{array}{c}\text { Fault } \\
\text { Detection }\end{array}$ & $\begin{array}{c}\text { Fault } \\
\text { Prediction }\end{array}$ & $\begin{array}{c}\text { Auto } \\
\text { Configuration }\end{array}$ & Reinstallation & & & & \\
\hline SHelp [4] & $\checkmark$ & $x$ & $x$ & $x$ & $x$ & $x$ & $\mathrm{x}$ & Fault Detection Rate \\
\hline SH-SLA [5] & $\checkmark$ & $x$ & $x$ & $x$ & $x$ & $x$ & $x$ & Fault Detection Rate \\
\hline SHF [6] & $\checkmark$ & $\checkmark$ & $x$ & $x$ & $x$ & $x$ & $x$ & Throughput \\
\hline SHP [7] & $\checkmark$ & $x$ & $x$ & $x$ & $x$ & $x$ & $x$ & Execution Time \\
\hline SHMR [9] & $\checkmark$ & $\checkmark$ & $x$ & $x$ & $x$ & $x$ & $x$ & Resource Utilization \\
\hline SFWA [10] & $\checkmark$ & $x$ & $x$ & $x$ & $x$ & $x$ & $x$ & SLA Violation Rate and Waiting Time \\
\hline MASA [14] & $\checkmark$ & $x$ & $x$ & $x$ & $x$ & $x$ & $x$ & Fault Detection Rate \\
\hline QoS-SC [15] & $x$ & $x$ & $\checkmark$ & $x$ & $x$ & $x$ & $x$ & $\begin{array}{c}\text { SLA Violation Rate and Resource } \\
\text { Utilization }\end{array}$ \\
\hline $\mathrm{ARC}[16]$ & $x$ & $x$ & $\checkmark$ & $x$ & $x$ & $x$ & $x$ & $\begin{array}{c}\text { SLA Violation Rate and Resource } \\
\text { Utilization }\end{array}$ \\
\hline SCP [17] & $x$ & $x$ & $\checkmark$ & $x$ & $x$ & $x$ & $x$ & Execution Time \\
\hline EASC [21] & $x$ & $x$ & $\checkmark$ & $\checkmark$ & $x$ & $x$ & $x$ & Energy Consumption \\
\hline AROMA [22] & $x$ & $x$ & $x$ & $\checkmark$ & $x$ & $x$ & $x$ & Resource Utilization \\
\hline COCCUS [23] & $x$ & $x$ & $x$ & $\checkmark$ & $x$ & $x$ & $x$ & Execution Time and Cost \\
\hline GOASC [24] & $x$ & $x$ & $x$ & $\checkmark$ & $x$ & $x$ & $x$ & Reliability and Resource Utilization \\
\hline CoTuner [25] & $x$ & $x$ & $\checkmark$ & $\checkmark$ & $x$ & $x$ & $x$ & Resource Utilization \\
\hline $\begin{array}{l}\text { RADAR (Our } \\
\text { Proposed } \\
\text { Technique) }\end{array}$ & $\checkmark$ & $\checkmark$ & $\checkmark$ & $\checkmark$ & $\checkmark$ & $\checkmark$ & $\checkmark$ & $\begin{array}{c}\text { Fault Detection Rate, Waiting Time, } \\
\text { Execution Time, Energy } \\
\text { Consumption, Throughput, Reliability, } \\
\text { Availability, Resource Contention, } \\
\text { SLA Violation Rate, Execution Cost, } \\
\text { Turnaround Time and Resource } \\
\text { Utilization } \\
\end{array}$ \\
\hline
\end{tabular}




\section{RADAR: Proposed Technique for Self-configuring and Self-healing of Cloud Resources}

Figure 1 shows the architecture of RADAR, which provides self-healing by handling unexpected failures and self-configuration of resources and applications for improving utilization of resources and reducing human intervention. SLA is used to describe the QoS parameters for execution of workloads using RADAR. RADAR is an autonomic resource management technique, which ensures to serve a huge number of requests without SLA violation and manages the cloud resources dynamically.

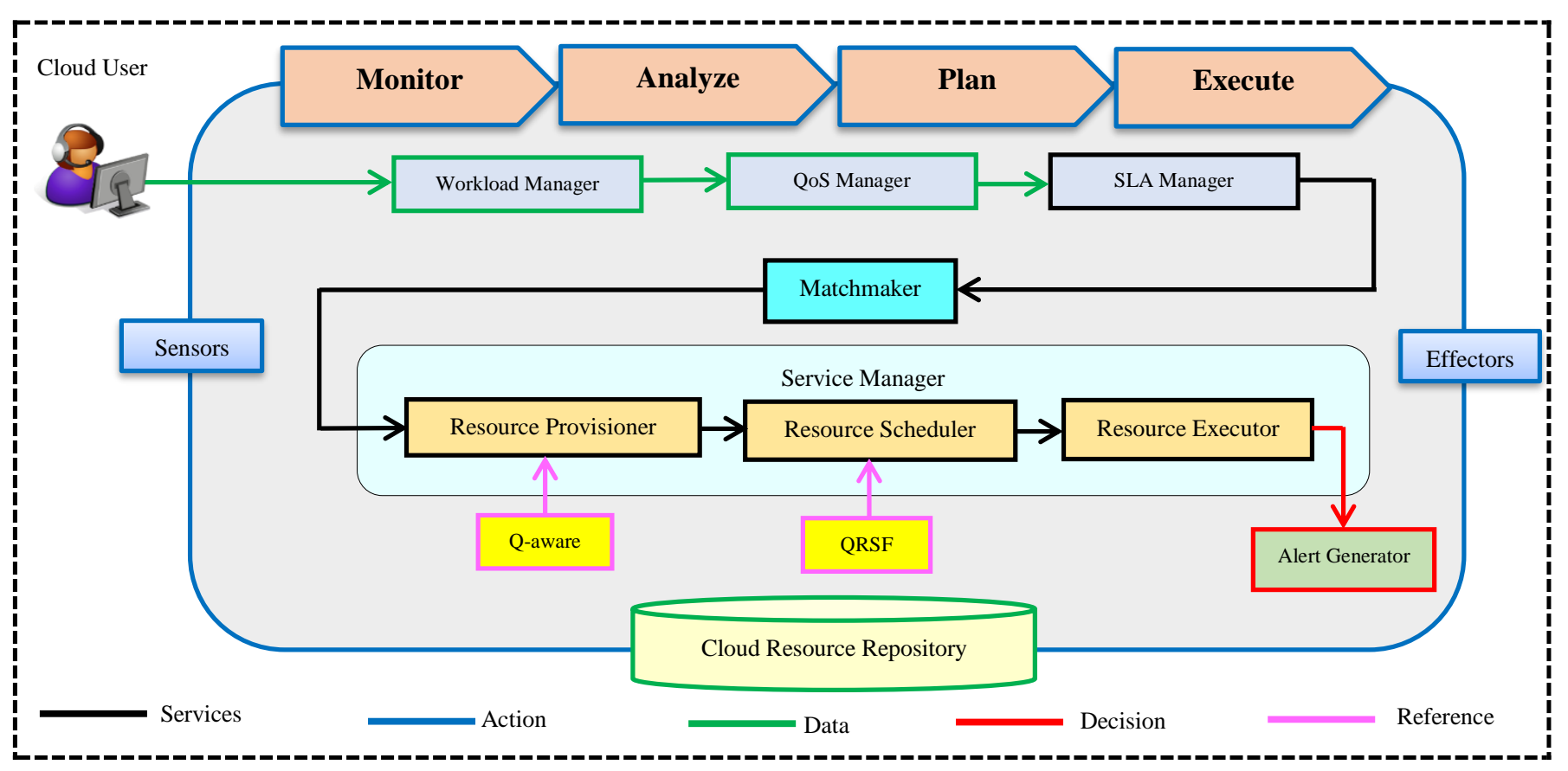

Figure 1: RADAR Architecture

For an execution of cloud-based applications, the mapping of cloud workloads to appropriate resources is found to be an optimization problem. Mathematically, the problem of resource scheduling for workload execution can be expressed as: a set of independent workloads $\left\{w_{1}, w_{2}, w_{3}, \ldots, w_{m}\right\}$ mapped on a set of cloud resources $\left\{r_{1}, r_{2}, r_{3}, \ldots, r_{n}\right\}$. For the continuous problem, $\mathrm{R}=\left\{r_{k} \mid 1 \leq \mathrm{k}\right.$ $\leq \mathrm{n}\}$ is a resource set and where $n$ is the total number of resources, while $\mathrm{W}=\left\{w_{i} \mid 1 \leq \mathrm{i} \leq \mathrm{m}\right\}$ is a workload set and where $m$ is the total number of cloud workloads. RADAR comprises of following units:

\subsection{Workload Manager}

To identify its QoS requirements of a workload, workload manager looks at different characteristics of that workload. Workload manager consists of three subunits: workload queue, workload description and bulk of workloads as shown in Figure 2. The Bulk of workloads are those which are submitted by users for execution. The QoS requirements and user constraints such as deadline and budget are described as workload description. Table 2 lists the various types of workloads and their QoS requirements [3] [26] [29], which are considered for evaluation.

Table 2: Cloud Workloads and their Key QoS Requirements

\begin{tabular}{|l|l|}
\hline \multicolumn{1}{|c|}{ Workload Name } & \multicolumn{1}{c|}{ QoS Requirements } \\
\hline Technological Computing & \multicolumn{1}{c|}{} \\
\hline Websites & Computing capacity \\
\hline E-Commerce & High availability, High network bandwidth and Reliable storage \\
\hline Graphics Oriented & Customizability and Variable computing load \\
\hline Backup and Storage Services & Visibility, Data backup, Latency and Network bandwidth \\
\hline Endeavour Software ${ }^{2}$ & Persistence and Reliability \\
\hline Productivity Applications ${ }^{3}$ & Correctness, Customer confidence Level, High availability and Security \\
\hline Critical Internet Applications $^{4}$ & Security, Data backup, Latency and Network bandwidth \\
\hline Mobile Computing Services & Usability, Serviceability and High availability \\
\hline Software/Project Development and Testing & Portability, Reliability and High availability \\
\hline Central Financial Services & Testing time, Flexibility and User self-service rate \\
\hline Online Transaction Processing & Integrity, Changeability, High availability and Security \\
\hline Performance Testing & Usability, Internet accessibility, High availability and Security \\
\hline
\end{tabular}

${ }^{1}$ Technological Computing: It contains numerical computations, atmospheric modeling and bioinformatics.

${ }^{2}$ Endeavour Software: It contains enterprise content management, SAP (System Application and Product) and email servers.

${ }^{3}$ Productivity Applications: It contains word editors and users signing up for emails.

${ }^{4}$ Critical Internet Applications: It contains web applications including the huge amount of scripting languages. 


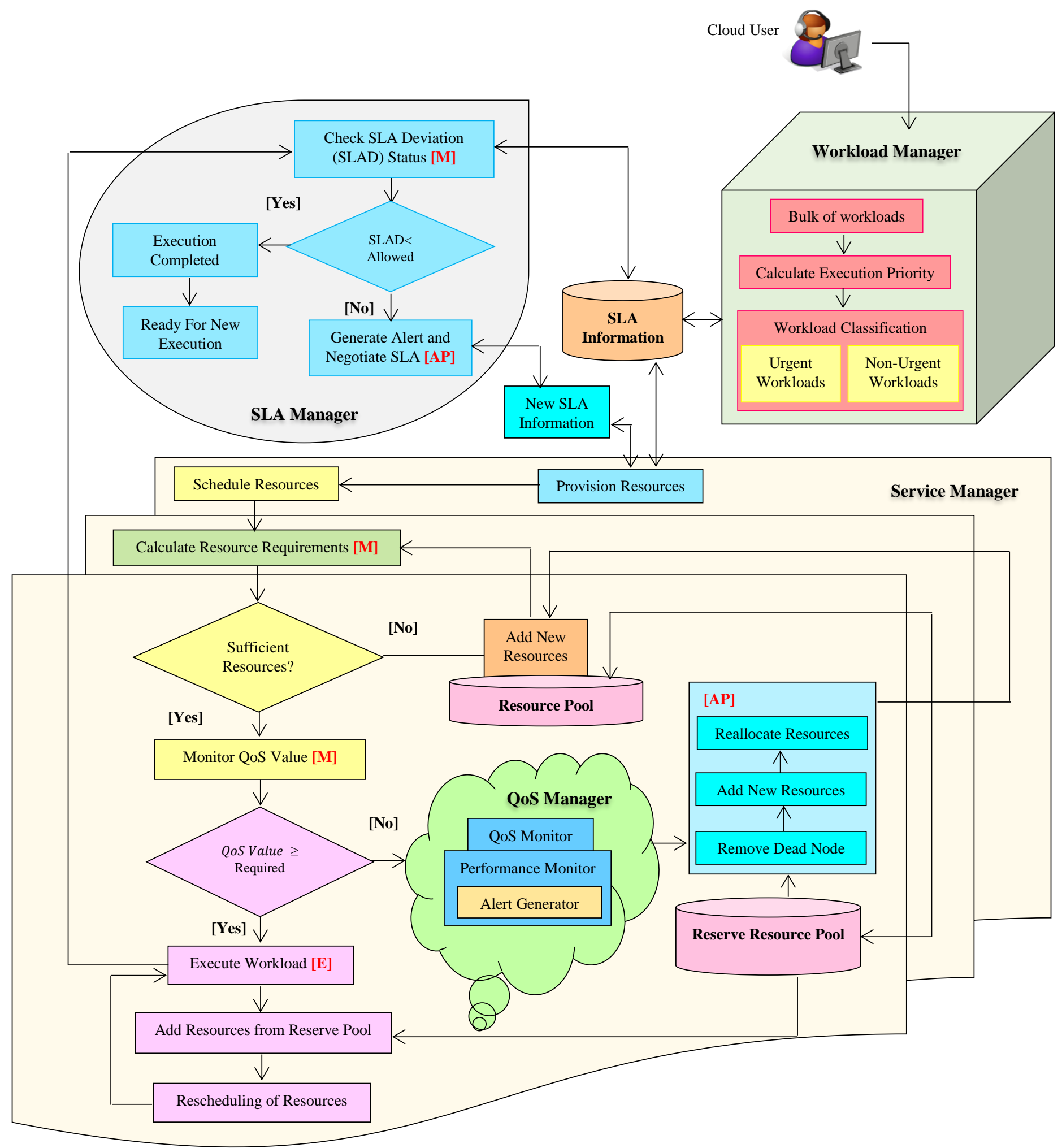

Figure 2: Working of RADAR

All the feasible user workloads are stored in a workload queue for provisioning of resources before actual resource scheduling [29]. Further, $K$-means based clustering algorithm is used for clustering the workloads for execution on the different set of resources [27]. The final set of workloads that we have chosen for evaluation is shown in Table 3.

Table 3: K-Means based Clustering of Workloads

\begin{tabular}{|c|c|c|}
\hline Cluster & Cluster Name & Workloads \\
\hline C1 & Compute & Performance Testing and Technical Computing \\
\hline C2 & Storage & Backup and Storage Services and E-commerce \\
\hline C3 & Communication & Mobile Computing Services, Critical Internet Applications and Websites \\
\hline C4 & Administration & $\begin{array}{c}\text { Graphics Oriented, Software/Project Development and Testing, Productivity Applications, } \\
\text { Central Financial Services, Online Transaction Processing and Endeavour Software }\end{array}$ \\
\hline
\end{tabular}

Workload manager decides the type of workload after submission based on their priority: Non-QoS (non-critical workloads) or QoS-oriented workloads (critical workloads). The priority of execution of workload is calculated based on their deadline. 


\subsection{QoS Manager}

Based on their QoS requirements, QoS Manager puts the workloads into non-critical (non-urgent workloads) and critical queues (urgent workloads) [2] [26] as shown in Figure 2. For critical workloads, it calculates the expected execution time of a workload and identifies the completion time of a workload, which is an addition of an execution time and waiting time [3]. The expected execution time of the workloads can be derived from workload task length or historical trace data [26]. All the QoS oriented workloads are put into the critical queue and sorted based on their priority decided by QoS Manager. The resources are provisioned immediately with the available resources, if completion time is lesser than desired deadline [29]. Otherwise, SLA is negotiated again for the extra requirement of resources and get required resources from the reserved pool for workload execution as shown in Figure 2. A penalty will apply in case of not fulfilling the deadline of critical workloads.

For non-critical workloads, the QoS manager checks whether the resources are free for execution. If required resources are available then workload will be executed directly, otherwise put workload into a waiting queue of non-critical workloads. If there is no condition (more requirement of resources and urgency), then use available resources to execute workload immediately, otherwise (if required resources are lower than the provided resources) put that workload into an under-scheduling state (workload in waiting state due to unavailability of resources) till the availability of required resources.

\subsubsection{QoS based Metrics}

The following metrics [2] [3] [26] [27] [29] [35] are used to calculate the value of QoS parameters such as reliability, availability, execution time, energy consumption, throughput, waiting time, resource contention, fault detection rate, resource utilization, turnaround time, execution cost and SLA violation rate.

Resource Utilization is a ratio of an execution time of a workload executed by a particular resource to the total uptime of that resource. The total uptime of resource is the amount of time available with a cloud resource set for execution of workloads. We have designed the following formula to calculate resource utilization $(R u)$ [Eq. 1].

$$
R u=\sum_{i=1}^{n}\left(\frac{\text { execution time of a workload executed on } i^{\text {th }} \text { resource }}{\text { total uptime of } i^{\text {th }} \text { resource }}\right)
$$

Where $n$ is the number of resources.

Energy Consumption: The energy model is developed on the basis that resource utilization has a linear relationship with energy consumption [35]. Energy Consumption (ENCN) of resources can be expressed as [Eq. 2]:

$$
E N C N=E N C N_{\text {Processor }}+E N C N_{\text {Transceivers }}+E N C N_{\text {Memory }}+E N C N_{\text {Extra }}
$$

$E N C N_{\text {Processor }}$ represents the processor's energy consumption, $E N C N_{\text {Transceivers }}$ represents the energy consumption of all the switching equipment. $E N C N_{\text {Memory }}$ represents the energy consumption of the storage device. $E N C N_{E x t r a}$ represents the energy consumption of other parts, including fans, the current conversion loss and others. For a resource $r_{k}$ at given time $t$, the resource utilization $R E S U_{t, k}$ is defined as [Eq. 3]:

$$
R E S U_{t, k}=\sum_{i=1}^{m} r u_{t, k, i}
$$

where $m$ is the number of cloud workloads running at time $t, r u_{t, k, i}$ is the resource usage of workload $w_{i}$ on resource $r_{k}$ at given time $t$. The actual energy consumption $\left(E_{c o n}\right)$ is $E C O N_{t, k}$ of a resource $r_{k}$ at given time $t$ is defined as [Eq. 4]:

$$
E_{\text {con }}=E C O N_{t, k}=\left(E N C N_{\text {max }}-E N C N_{\text {min }}\right) \times R E S U_{t, k}+E N C N_{\text {min }}
$$

where $E N C N_{\max }$ is the energy consumption at the peak load (or $100 \%$ utilization) and $E N C N_{\min }$ is the minimum energy consumption in the active/idle mode (or as low as 1\% utilization), which can be calculated using [Eq. 2] .

Execution Cost $\left(E_{\text {cost }}\right)$ : It is the minimum cost spend to execute workload and measured in terms of Cloud Dollars (C\$) and is defined as [Eq. 5]:

$$
E_{\text {cost }}=\min \left(c\left(r_{k}, w_{i}\right)\right) \text { for } 1 \leq k \leq n \text { and } 1 \leq i \leq m
$$

where $c\left(r_{k}, w_{i}\right)$ is the cost of workload $w_{i}$ which executes on resource $r_{k}$ as defined below:

$$
\mathrm{c}\left(r_{k}, w_{i}\right)=\frac{\text { completion }\left(w_{i}, r_{k}\right)}{\text { completion }_{m}\left(w_{i}\right)}+\text { Penalty Cost }
$$

completion $_{m}\left(w_{i}\right)$ denotes the maximal completion time of the cloud workload. Before estimation of the execution time, completion time of a resource should be defined as:

$$
\text { completion }_{m}\left(w_{i}\right)=\max _{r_{k} \in R} \text { completion }\left(w_{i}, r_{k}\right)
$$


Completion time or completion $\left(w_{i}, r_{k}\right)$ is defined as the time in which a resource can finish the execution of all the previous workloads in addition to the execution of workload $w_{i}$ on resource $r_{k}$ is defined as:

$$
\text { completion }\left(w_{i}, r_{k}\right)=\text { available_time } r_{k} \pm P T C_{m}\left(w_{i}\right)
$$

where

$$
\operatorname{PTC}_{m}\left(w_{i}\right)=\max _{r_{k} \in R} \operatorname{PTC}\left(w_{i}, r_{k}\right)
$$

where $m$ is the number of workloads. available_time $r_{k}$ is the switching time to transfer workload from a waiting queue to ready queue for execution on resource $r_{k}$. Penalty Cost is defined as an addition to penalty cost for different workloads (if applicable).

$$
\text { Penalty Cost }=\sum_{i=1}^{C}\left(P C_{i}\right)
$$

Delay time is defined as the time difference between expected completion time and actual completion time. It is expressed as

$$
\text { Delay Time }=\text { Expected Completion Time }- \text { Actual Completion Time }
$$

$$
P C=\left\{\begin{aligned}
\text { Penalty }_{\text {minimum }}, & \text { if Expected Completion Time } \geq \text { Actual Completion Time } \\
\text { Penalty }_{\text {minimum }}+[\text { Penalty Rate } \times \mid \text { Delay Time } \mid], & \text { if Expected Completion Time }<\text { Actual Completion Time }
\end{aligned}\right.
$$

Where $c \in C, C$ is the set of penalty cost with different levels specified in RADAR.

Execution Time $\left(E_{\text {time }}\right)$ : It is the finishing time $L_{w}$ of the latest workload and can also be represented as PTC workload $w_{i}$ on resource $r_{k}$.

$$
E_{\text {time }}=\min \left(L_{w_{i}}\right) w_{i} \in W
$$

The value of [Number of workloads $\times$ number on resources] for every workload on resources is calculated from Predictable Time to Compute (PTC) matrix [35]. Columns of PTC matrix demonstrate the estimated execution time for a specific resource while rows on PTC matrix demonstrate the execution time of a workload on every resource. In this research work, the PTC benchmark simulation model is used, which has been introduced in [34] to address the problem of resource scheduling. The expected execution time of the workloads can be derived from workload task length or historical trace data. A high variation in execution time of the same workload is generated using the gamma distribution method. In the gamma distribution method, a mean workload execution time and coefficient of variation are used to generate PTC matrix [36]. Table 4 shows a $10 \times 6$ subset of the PTC matrix and results provided in this research work used the matrix of size $90 \times 36$.

Table 4: A $10 \times 6$ Subset of the PTC Matrix

\begin{tabular}{|c|c|c|c|c|c|c|}
\hline Workloads & $\boldsymbol{r}_{\mathbf{1}}$ & $\boldsymbol{r}_{\mathbf{2}}$ & $\boldsymbol{r}_{\mathbf{3}}$ & $\boldsymbol{r}_{\mathbf{4}}$ & $\boldsymbol{r}_{\mathbf{5}}$ & $\boldsymbol{r}_{\mathbf{6}}$ \\
\hline $\boldsymbol{w}_{\mathbf{1}}$ & 112.14 & 141.44 & 136.65 & 109.66 & 170.46 & 137.58 \\
\hline $\boldsymbol{w}_{\mathbf{2}}$ & 152.61 & 178.26 & 149.78 & 114.26 & 198.92 & 148.69 \\
\hline $\boldsymbol{w}_{\mathbf{3}}$ & 147.23 & 190.23 & 180.26 & 121.65 & 141.65 & 152.69 \\
\hline $\boldsymbol{w}_{\mathbf{4}}$ & 103.62 & 159.63 & 192.85 & 107.69 & 139.89 & 139.36 \\
\hline $\boldsymbol{w}_{\mathbf{5}}$ & 178.65 & 171.35 & 201.05 & 127.65 & 169.36 & 201.66 \\
\hline $\boldsymbol{w}_{\mathbf{6}}$ & 193.62 & 142.65 & 205.36 & 132.26 & 188.33 & 207.72 \\
\hline $\boldsymbol{w}_{\mathbf{7}}$ & 187.24 & 138.23 & 217.58 & 147.69 & 112.39 & 210.98 \\
\hline $\boldsymbol{w}_{\mathbf{8}}$ & 124.13 & 110.65 & 212.39 & 141.26 & 135.88 & 169.35 \\
\hline $\boldsymbol{w}_{\mathbf{9}}$ & 138.56 & 123.65 & 170.26 & 181.65 & 116.61 & 142.87 \\
\hline $\boldsymbol{w}_{\mathbf{1 0}}$ & 131.29 & 129.65 & 142.69 & 199.34 & 125.36 & 147.69 \\
\hline
\end{tabular}

The columns of PTC matrix demonstrate the estimated execution time for a specific resource while rows on PTC matrix demonstrate the execution time of a workload on every resource.

Resource Contention: It occurs when the same resource is shared by more than one workload [29]. The main reasons for resource contention are: i) when the same resource is used to execute more than one workload, ii) unavailability of required number of resources to execute current set of workloads, and iii) the number of workloads with urgent deadline, which are trying to access the required same resources; more workloads with urgent deadline creates more resource contention. Resource contention (ResCon) is defined during scheduling of resources at time $\boldsymbol{t}$ and is calculated as

$$
\begin{aligned}
& \operatorname{ResCon}(t)=\sum_{r \in \text { ResourceList }} \operatorname{ResCon}(t, r) \\
& \operatorname{ResCon}(t, r)=\sum_{r t \in \text { ResourceType }} \operatorname{ResCon}(t, r, r t)
\end{aligned}
$$

where $\boldsymbol{r}$ is the list of resources, $\boldsymbol{r t}$ specifies the type of resource (overloaded or not). We have considered $W_{Q}$ as a set of total workloads (Eq. 16), which is executed by different resources. 


$$
W_{Q}=\left\{W_{1}, W_{2}, \ldots \ldots \ldots \ldots \ldots W_{m}\right\}
$$

During execution of workloads, some workloads overload the resources, which is denoted by a set called $O V E R L O A D$ (Eq. 17).

$$
\text { OVERLOAD }=\left\{W_{1}, W_{2}, \ldots \ldots \ldots \ldots . . . W_{o}\right\}
$$

RCStatus $(t, r, r t)$ specify the current status of resource contention (Eq. 18) in terms of Boolean statements i.e. True or False [29].

$$
\text { RCStatus }(t, r, r t)= \begin{cases}1, & \sum_{w \in W_{Q}(t, r)}(r t \in w . \text { OVERLOAD }==\text { TRUE } ? 1: 0) \geq 1 \\ 0, & \text { otherwise }\end{cases}
$$

Where, $w$. OVERLOAD specifies the set of workloads, which overloads the resource at time $t$. (rt $\in$ w.OVERLOAD $==$ $T R U E$ ? 1:0) finds the status of a resource, which is overloaded or not. If its value is equal to or more than one, then the value of RCStatus $(t, r, r t)$ is one, otherwise, its value is zero.

$$
\operatorname{ResCon}(t, r, r t)= \begin{cases}\sum_{w \in W_{Q}(t, r) \wedge r t \in w . O V E R L O A D} w . \text { ResourceRequirment }[r t], & \text { RCStatus }(t, r, r t)=1 \\ 0, & \text { otherwise }\end{cases}
$$

w. ResourceRequirment specifies the resource requirement of $\boldsymbol{w}$ in terms of capacity (storage, processor or memory) and throughout all experiments this value, measured in seconds, is as a value for comparison, and not an exact time for resource contention.

SLA violation Rate is defined as the product of Failure rate and weight of SLA [3] and is calculated as:

$$
\begin{aligned}
& \text { List of SLA }=\left\langle m_{1}, m_{2} \ldots \ldots \ldots \ldots \ldots m_{n}>\text {, where } n\right. \text { is the total number of SLAs. } \\
& \text { Failure }(m)= \begin{cases}1, & m \text { is not violated } \\
0, & m \text { is violated, }\end{cases}
\end{aligned}
$$

Failure rate (Eq. 21) is computed as a ratio of the summation of all the SLA violated to the total number of SLAs.

$$
\text { Failure Rate }=\sum_{i=1}^{n}\left(\frac{\text { Failure }\left(m_{i}\right)}{n}\right)
$$

SLA violation rate is a product of failure rate and summation of every weight for every SLA

$$
\text { SLA Violation Rate }=\text { Failure Rate } \times \sum_{i=1}^{n}\left(w_{i}\right)
$$

Where $w_{i}$ is the weight for every SLA, which is calculated using (Eq. 23). The consequence of collected data is used by the following formula [26] to calculate the weight of quality attributes (Eq. 23):

$$
w_{i}=\frac{1}{N_{f} \times\left(M_{v}+q\right)} \times \sum_{a=1}^{N_{f}} R_{a} \times 100
$$

Where, $i$ is cloud workload, $q$ is level of measurement of quality attribute (q-value), $N_{f}$ is number of research papers used to collect data, $M_{v}$ is maximum value for a quality attribute and $R_{a}$ is sum of responses for an attribute; the value of $w_{i}$ will be in the range $0 \%-100 \%$. An analysis has been conducted to acquire the data from different research papers of cloud computing from reputed journals about cloud workloads with the objective to know that how to assign the weights to the quality attributes according to significance [26]. Subsequently receiving the responses, an industry standard baseline and adequate weights to the quality attributes have been defined. The conversion metric is used to assign the values (minimum $=1$ and maximum $=5$ ) corresponding to the percentage [27] as shown in Table 5.

Table 5: Conversion Metric

\begin{tabular}{|c|c|}
\hline Approximate Weight (\%) & Weight \\
\hline $0-20$ & 1 \\
\hline $20-40$ & 2 \\
\hline $40-60$ & 3 \\
\hline $60-80$ & 4 \\
\hline $80-100$ & 5 \\
\hline
\end{tabular}

Table 6: Level of Measurement of Quality Attribute

\begin{tabular}{|c|c|}
\hline Level of Measurement of Quality Attribute & q-value \\
\hline High & 3 \\
\hline Medium & 2 \\
\hline Low & 1 \\
\hline
\end{tabular}


The level of measurement of a quality attribute will be of three types: High, Medium and Low as described in Table 6. The result of the data analysis is as follows; the number of research papers of different contexts have been studied and maximum probable value for a quality attribute is 5. For example, computing the average of "Availability" quality attribute under workload "Websites" is as follows:

$N_{f}=11$, Workload = Website and Quality Attribute $=$ Availability, $M_{v}=5, q=3$ (because availability should be high described in QoS requirements of SLA and q-value has been calculated with the help of QoS metrics) and sum of the responses $\sum_{k=1}^{11} R_{a}=29$

$$
w_{i}=\frac{1}{11 \times(5+3)} \times 29 \times 100=32.95
$$

For $w_{i}=32.95$, the average weight assigned for availability is 2 by using Table 5. Through this technique [26] [27] the average weights for every quality attribute have been calculated.

Fault Detection Rate is the ratio of number of faults detected to the total number of faults existing. Fault Detection Rate (FDR) is calculated as

$$
F D R=\frac{\text { Number of Faults Detected }}{\text { Total number of Faults }}
$$

Faults can be a network, software or hardware, which is detected based on the violation of SLA.

Throughput is the ratio of the total number of workloads to the total amount of time required to execute the workloads and it is calculated as

$$
\text { Throughput }=\frac{\text { Total Number of Workloads }\left(W_{n}\right)}{\text { Total amount of time required to execute the workloads }\left(W_{n}\right)}
$$

Reliability of the resource has to be checked for scheduling of the resources [35]. With the help of reliability parameter, we can check the fault tolerance of the resource. Reliability of the resource is calculated as:

$$
r e=e^{-\lambda \mathrm{t}}
$$

$r e=$ reliability of resource, $t=$ time for the resource to deal with its request for any workload's execution and $\lambda=$ the failure rate of the resource at the given time, which is calculated using [Eq. 21].

Availability is defined as an interval of the real line. Availability is represented as

$$
A=\sum_{t=0}^{c} A(t)
$$

where $c$ is an arbitrary constant (c>0) and its value is chosen to select the time interval, for which the availability of system can be tested. We have used $c=850$ seconds for Test Case 4 to find the experiment statistics (Section 4$)$. Therefore, the availability $A(t)$ at time $t>0$ is represented by

$$
A(t)=\operatorname{Pr}[X(t)=1]=E[X(t)=1]
$$

Further, the status function is defined as:

$$
X(t)= \begin{cases}1, & \text { RADAR functions at time } t \\ 0, & \text { Otherwise }\end{cases}
$$

Average Waiting Time or Waiting Time is a ratio of the interval computed between workload execution start time (WE $\left.E_{i}\right)$ and workload submission time $\left(W S_{i}\right)$ to the number of workloads. It is calculated as

$$
W(n)=\sum_{i=1}^{n}\left(\frac{W E_{i}-W S_{i}}{n}\right)
$$

where $n$ is the number of workloads.

Turnaround Time is a ratio of the interval computed between workload completion time $\left(W C_{i}\right)$ and workload submission time $\left(W S_{i}\right)$ to the total number of workloads. It is calculated as

where $n$ is the number of workloads.

$$
T(n)=\sum_{i=1}^{n}\left(\frac{W C_{i}-W S_{i}}{\mathrm{n}}\right)
$$




\subsection{SLA Manager}

An SLA document is prepared based on SLA information finalized between user and provider. SLA document contains the information about SLA Violation (the value of minimum and maximum deviation and compensation or penalty rate in case of violation of SLA). Deviation status estimates the deviation of QoS from predictable values [3]. A penalty will be imposed, if the deviation is more than the allowed for urgent workloads and penalty can be an allocation of reserve resources to a specific workload for compensation. RADAR minimizes the effect of inaccuracy by: 1) adding slack time during scheduling and 2) considering the penalty-compensation clause in SLAs in case of its violation. SLA manager of RADAR tries to execute the workload within userspecified deadline and budget with maximum resource utilization and without violation of SLA.

Based on the workload deadline, workloads are classified into two categories: i) critical and ii) non-critical. Table 7 describes the details about Non-Critical (Relaxed Deadline) and Critical (Urgent Deadline) workloads and the calculation of compensation and penalty [29].

Table 7: Types of Workload and their Urgency Details

\begin{tabular}{|c|c|c|c|c|c|}
\hline Workload Type & $\begin{array}{c}\text { Slack Time } \\
\text { (Seconds) }\end{array}$ & $\begin{array}{c}\text { Delay Time } \\
\text { (Seconds) }\end{array}$ & $\begin{array}{c}\text { Deviation } \\
\text { Status }\end{array}$ & $\begin{array}{c}\text { Minimum } \\
\text { Penalty }\end{array}$ & Penalty Rate \\
\hline \multirow{2}{*}{$\begin{array}{c}\text { Non-Critical (Relaxed } \\
\text { Deadline) }\end{array}$} & \multirow{2}{*}{60} & $0-50$ & $5 \%$ & 50 Seconds & $2 \%$ \\
\cline { 3 - 6 } & & $51-100$ & $10 \%$ & 100 Seconds & $3 \%$ \\
\cline { 3 - 6 } & \multirow{2}{*}{$\begin{array}{c}\text { Critical (Urgent } \\
\text { Deadline) }\end{array}$} & $101-150$ & $15 \%$ & 150 Seconds & $4 \%$ \\
\cline { 3 - 6 } & & $0-50$ & $5 \%$ & 200 Seconds & $5 \%$ \\
\cline { 3 - 6 } & & $51-100$ & $10 \%$ & 400 Seconds & $6 \%$ \\
\hline
\end{tabular}

The following example shows the estimation of penalty or compensation for "CRITICAL" workload with Delay Time $=50$ (Deviation Status $=5 \%$ ) seconds:

$$
\begin{aligned}
& \text { Compensation }=\text { Penalty }_{\text {minimum }}+[\text { Penalty Rate } \times \text { Delay Time }] \\
& \text { Compensation }=200 \text { Seconds }+[5 \times 50 \text { Seconds }]=450 \text { Seconds }
\end{aligned}
$$

It will provide 450 seconds free cloud service as a compensation or penalty.

\subsection{Service Manager}

Initially, submitted workloads are moved to workload queue $\left(W_{Q}=\left\{W_{1}, W_{2}\right.\right.$, $\left.\left.W_{m}\right\}\right)$ as an input. The Matchmaker maps the workloads to the suitable resources using Q-aware [26], which uses SLA information for resource provisioning. Q-aware [26] is a resource provisioning technique, in which resource provisioner provisions resources to different cloud workloads based on their QoS requirements as described in SLA. Further, resource scheduler of QoS based Resource Scheduling Framework (QRSF) schedules the provisioned resources [27] for workload execution. Figure 3 shows the interaction of cloud user, SLA manager and service manager for execution of workloads, in which SLA is signed initially. Based on SLA information, RADAR generates the workload schedule. A workload will be executed within its specified budget and deadline with maximum resource utilization and without violation of SLA. In case of the SLA violation, RADAR uses STAR [3] to renegotiate SLA again with new deadline and budget. Workload will be dispatched for execution after verification of every critical parameter and resource executor executes the workload after payment.

RADAR returns the resources to resource pool after successful execution of workloads. Finally, updated workload's execution information returns to the corresponding cloud user. Figure 2 shows the execution of workloads using subunits of an autonomic model [3] such as: Monitor [M], Analyze and Plan [AP] and Executor [E]. Performance monitor monitors the performance of workload execution continuously and generates alerts in case of degradation of performance. RADAR generates following alerts in two different cases as shown in Figure 2.

- Alert 1: Unavailability of required resources for workload execution then perform an Action 1 for Reallocation of resources.

- Alert 2: If the value of SLA deviation is more than allowed then perform an Action 2 to Renegotiate SLA.

RADAR performs the same action two times. The system is treated as down if RADAR fails to correct it. To interact with the outside environment, RADAR uses two interfaces of an autonomic model [3]: Sensors and Effectors. Sensors read the QoS value to check the performance of the system. Initially, coordinator node gets the updated information (QoS value, faults or new updates) from processing nodes and transfers this information to Monitors. For example: energy consumption of workload execution can be one reason for performance degradation. Sensors reads the value of consumption of energy and compare with its threshold value to test the performance of the system. RADAR continues the workload execution if the value of consumption of energy is less than its threshold value. Otherwise, add new resources to improve energy efficiency using these steps: i) declare the current node as dead, ii) eliminate the node, iii) add new resources and iv) start execution after reallocation of resources and send the updated information to the coordinator node [35] [37]. Effector exchanges the updated information about new alerts, rules and policies to the other nodes. Service Manager works in two phases: self-configuring and self-healing as shown in Figure 3. 


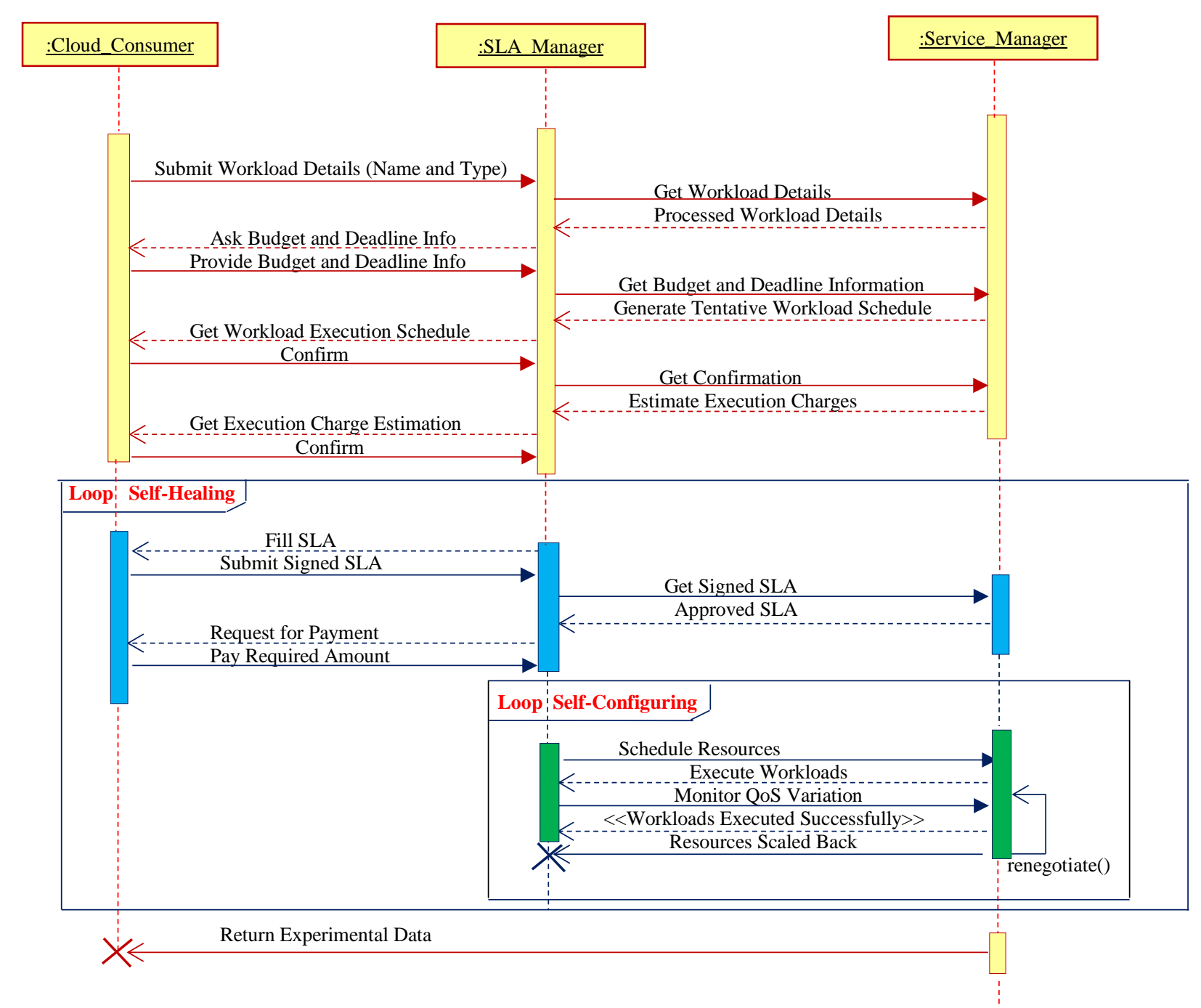

Figure 3: Design Description

\subsubsection{Self-Configuring}

Some components of the system need updates and other components need reinstallation due to changing conditions of a computing environment. Based on the generated alert, RADAR offers self-configuring of installation of outdated or missed components automatically. We propose an algorithm for self-configuring, which offers an autonomic installation of new components or reinstallation of outdated or missed components. [Algorithm 1: Self-Configuring] works in three different sub-modules: i) monitoring, ii) analyzing and planning and iii) executing. Figure 4 shows the graphical representation of pseudocode of an Algorithm 1 .

Initially, Monitor gathers the updated information (QoS value) from different sensors and monitors the performance variations continually by doing the comparison between actual and expected value of QoS. Basically, an expected value of QoS is considered as its threshold value, which also contains the maximum value of SLA deviation. We have observed the actual value of QoS based on SLA violation, new updates (missing or outdated components) and faults (hardware, software or network).

For Monitoring Module, Figure 4 shows that RADAR checks the status of active components in monitoring unit by using software and hardware component agent. Software component agent monitors the status of active software components, which can be 'MISSING' or 'OUTDATED'. If the status is 'MISSING', then software component agent reinstalls the component after uninstalling existing component. If the status is 'OUTDATED', then software component agent installs the new version of that component. Hardware component agent monitors the status of active hardware components and it uses the log information to track the status of different hardware components and generate an alert in case of error. Log information [29] has fields such as: 1) Time Stamp (Time of occurrence of error in that event), 2) Event Type (type of event occurred i.e. 'CRITICAL' OR 'ERROR'), 3) Source (Source is the software that logged the event, which can be either a program name, such as "SQL Server," or a component of the system or of a large program, such as a driver name) and 4) Event Id (Event has unique identity number). Alert will be generated if any of the event ('CRITICAL' OR 'ERROR') occurs and use log information [Component_Name and Compoenent_Id] to update the database.

A "machine checks log" is used in RADAR to manage the failures of hardware components and it can generate alert for internal errors quickly. RADAR uses a centralized database to maintain the machine check logs related to hardware. Further, lexical analyzer-based freeware tools such as MCat (windows) and MCELogs (Linux) [39] are used to refine the log information (Event 


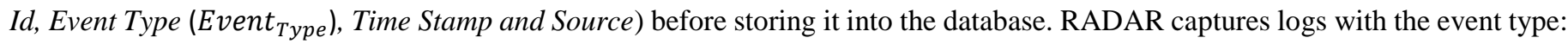
"CRITICAL" or "ERROR".

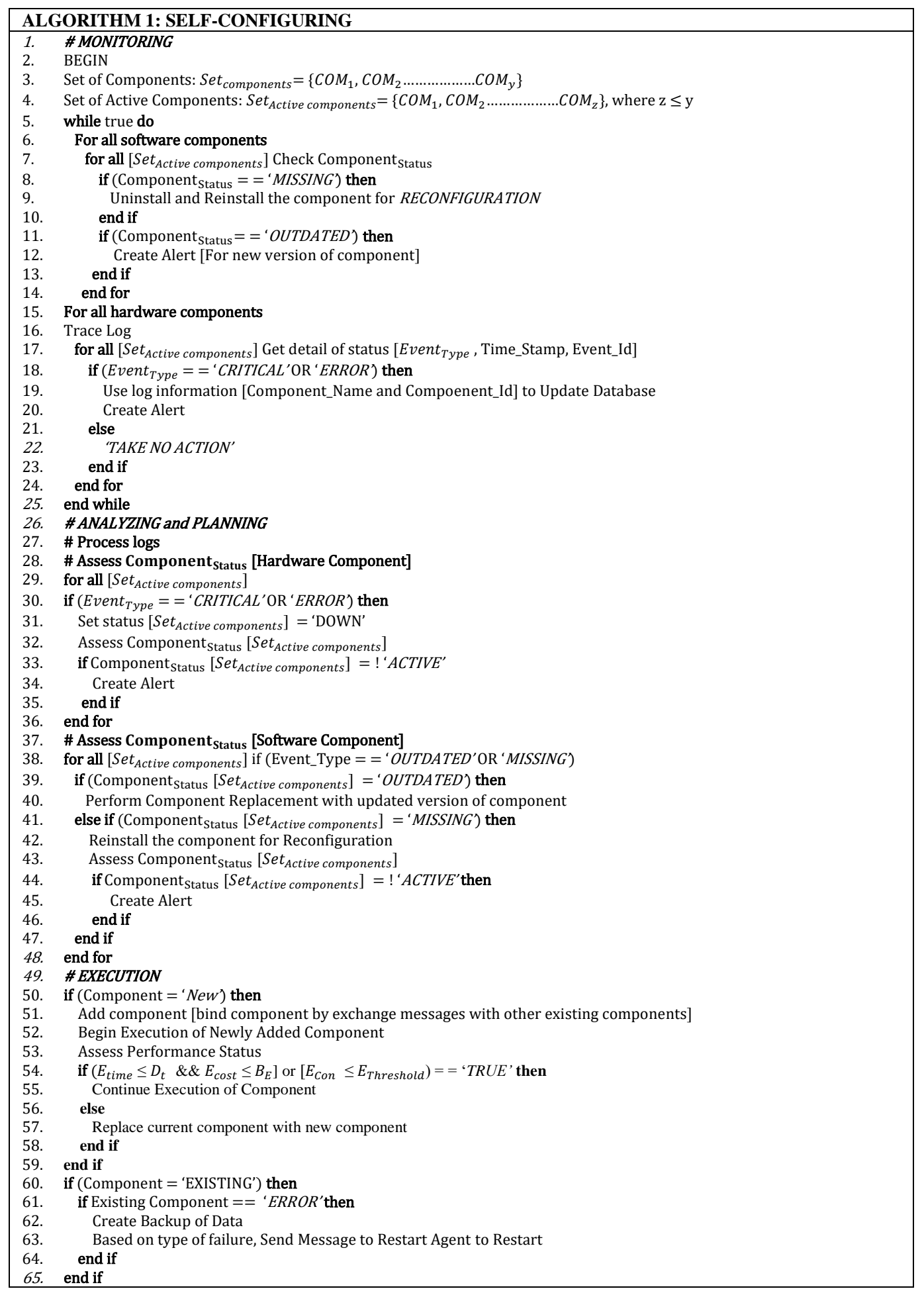

Analyzing and Planning Module analyzes the generated alert and behavior of software and hardware component. For hardware component, it declares the component as 'DOWN' if the status of a component is either 'CRITICAL' OR 'ERROR' and restarts it to its status again. If its status changes to 'ACTIVE', then continue execution, the otherwise 'INACTIVE' component will be replaced with a new component and start execution [29]. For software component, if its status is either 'MISSING' or 'OUTDATED' (Event Type) then software agent performs the following steps: 1) if Event Type is 'MISSING' then reinstall the component and 2) if Event Type is 'OUTDATED' then, that component is replaced with an updated version. After analysis of the status of hardware and software component, RADAR makes a plan to correct these errors automatically. Further, RADAR checks the status of active components $\left[\mathrm{Set}_{\text {Active components }}\right]$ continually for future performance analysis. 


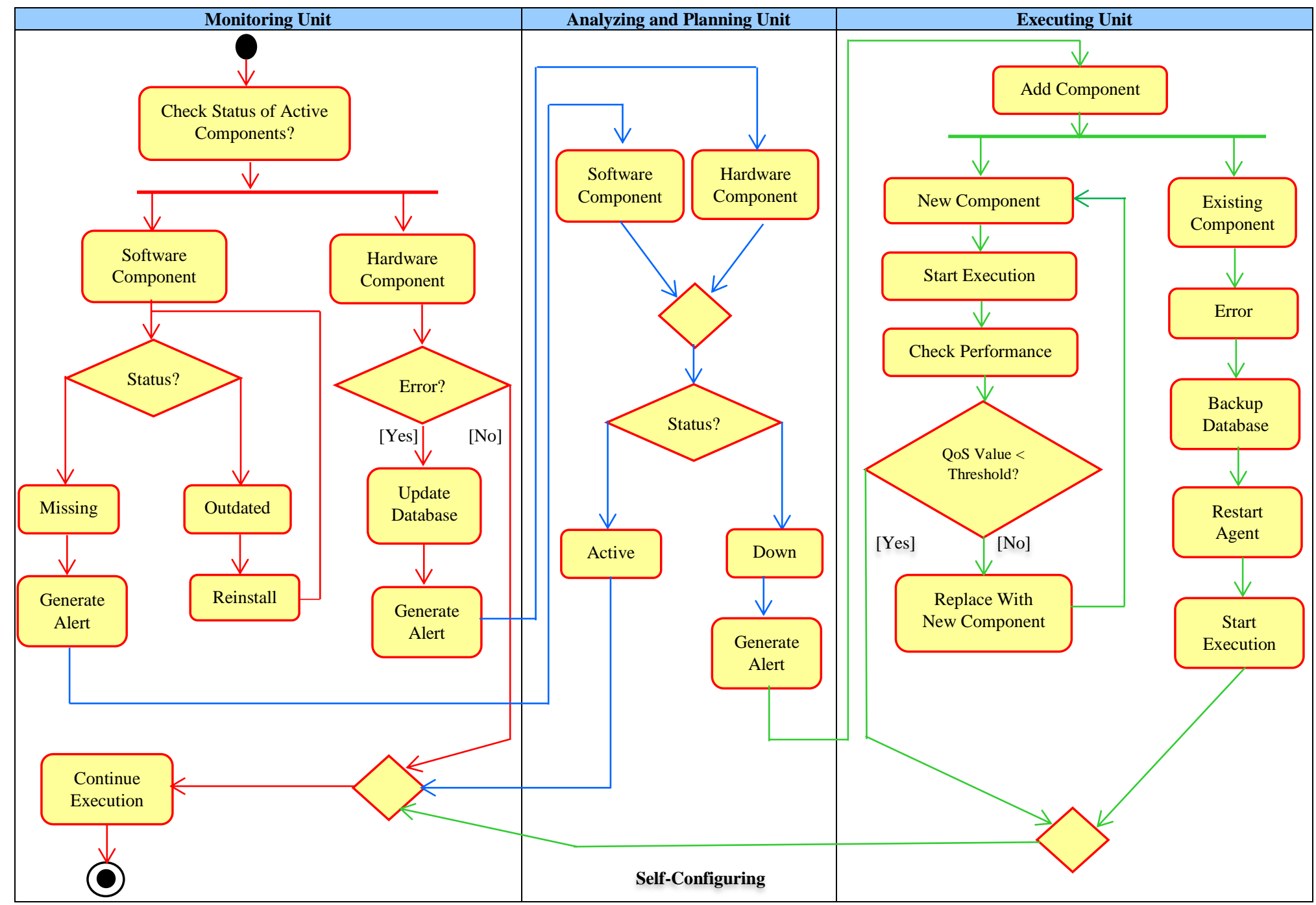

Figure 4: Process of Self-Configuring

For new component, Execution module implements the plan and binds the new component with existing components by exchanging messages between them. Further, it starts the execution of a new component as shown in Figure 4. During execution of workload, the value of Execution Time $\left(E_{\text {time }}\right)$, Average Cost $\left(E_{\text {cost }}\right)$ and Energy Consumption $\left(E_{\text {con }}\right)$ is calculated for every workload. If this condition ( $\left[E_{\text {time }} \leq D_{t} \& \& E_{\text {cost }} \leq B_{E}\right]$ or $\left[E_{\text {Con }} \leq E_{\text {Threshold }}\right]$ ), is false then generate an alert and replace this component with another qualified component.

$E_{\text {Threshold }}$ is maximum allowed value for energy consumption [27] [29]. Estimated Budget ( $\left.B_{E}\right)$ is the maximum value of cost that user wants to spend and measured in Cloud Dollars $(C \$)$. Deadline Time $(D t)$ is defined as the time duration between the current time $\left(C t_{i}\right)$ and workload deadline $\left(W d_{i}\right)$ and [Eq. 32] is used to calculate Deadline Time.

$$
\text { Deadline Time }\left(D t_{i}\right)=\sum_{i=1}^{n}\left(W d_{i}-C t_{i}\right)
$$

Where $W d_{i}$ is workload deadline and $C t_{i}$ is the current time. For existing component, restart the component after saving its state if an error occurs. If the component is still not performing effectively, then the issue can be resolved in two ways: a) install an updated version of a component or b) reinstall the component. Further, RADAR manages all the updates and stores in a centralized database and also maintains the backup of it as database replica. Backup Database (BD) can be used in future if master Data Base (DB) goes down [39] and BD acts as the master until the master database is up again.

\subsubsection{Self-Healing}

RADAR offers self-healing to ensure the proper functioning of a system by making mandatory changes to recover from the different types of faults, which can be a network, software or hardware fault. Network fault can occur due to network breakage, physical damage, packet loss and lack of scalability in distributed networks. Hardware fault can occur due to non-functioning of components such as hard disk, RAM or processor. Software fault can occur due to unavailability of a required number of resources, storage space, resource contention (deadlock) and unhandled exception in high resource intensive workloads. [Algorithm 2: Self-Healing] 
is working in three different sub-modules: i) monitoring, ii) analyzing and planning and iii) executing. Figure 5 shows the graphical representation of pseudocode of an Algorithm 2. Monitoring Module comprises of hardware, network and software agent to manage the different types of faults. Hardware agent continually monitors the performance of hardware components such as CPU and MEMORY.

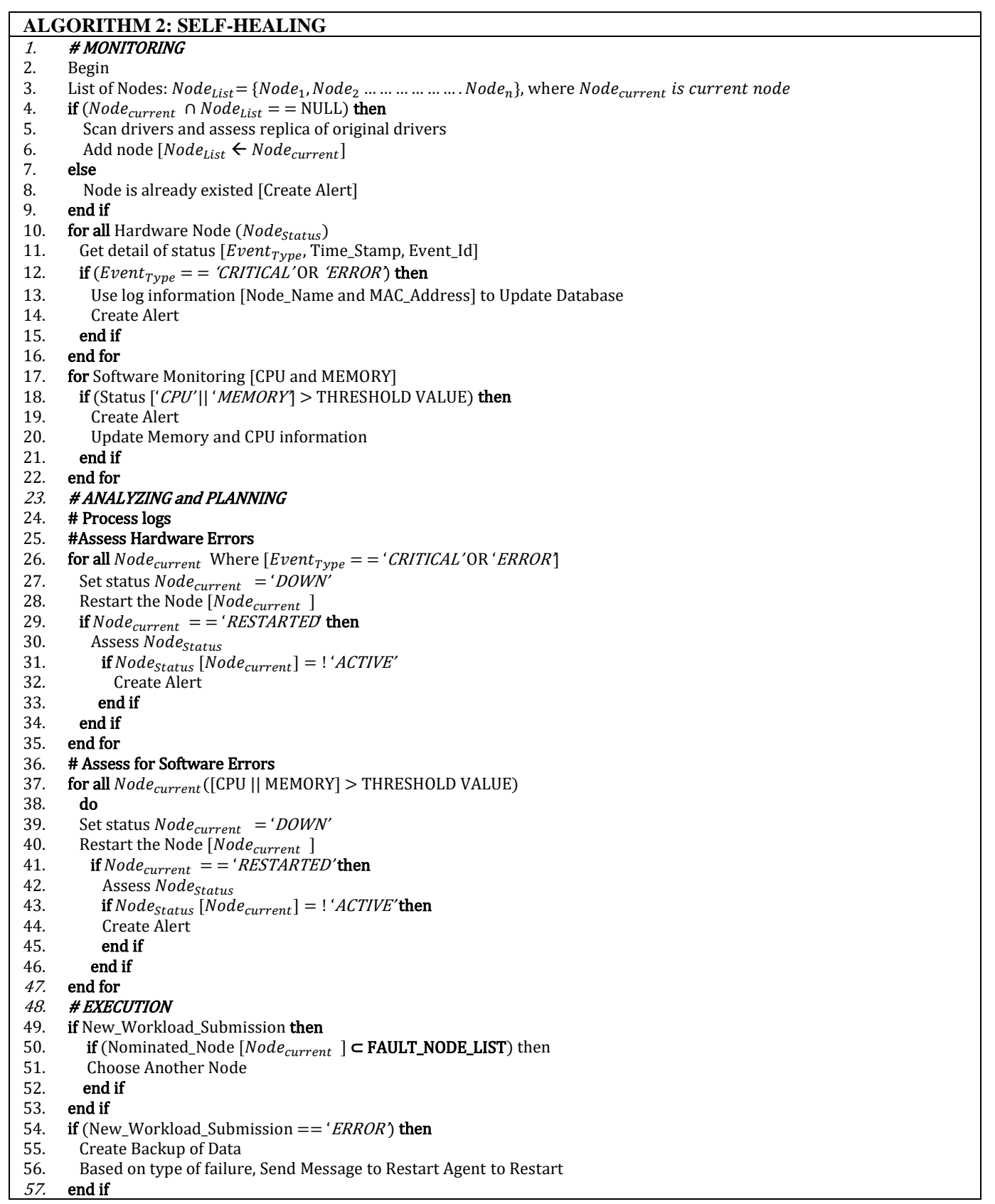

To maintain the performance of the system, RADAR performs hardening [32] to ensure that the driver is working properly. Hardware Hardening is the process in which the driver works correctly even though faults occur in the device that it controls or other faults originating from outside the device [32]. A hardened driver should not hang the system or allow the uncontrolled spread of corrupted data as a result of any such faults [39]. Whenever the node is added to the network, Hardware Hardening Agent (HHA) checks for its device drivers and perform the process of hardening. [ALGORITHM 3: Hardware Hardening Process (HHP)] shows the process of hardware hardening in RADAR.

Initially, HHA starts the process of hardening by scanning the drivers to be hardened [39]. Further, when the new node is added, a replica of the original drivers is created. RADAR uses the concept of carburizer [32], which pushes the code on that node when a new node is added. The process of hardware hardening consists of these consecutive steps: 1) scan the source code for all drivers, 2) find the chance of failure in code, 3) replace the code after identification of the code, 4) replace the original drivers with the hardened drivers after hardening, 5) hardware agent monitors the performance of hardened driver continuously and 6) replaces the hardened driver with original driver in case of performance degradation. RADAR uses machine check log to maintain the database of hardware failures. RADAR continually monitors the status of the system, which contains the information about the event [Event Type, Event Id, Timestamp] and event type which can be either 'CRITICAL' OR 'ERROR'. Further, hardware agent generates the alert and maintains the log information [Node_Name and MAC_Address] as shown in Figure 5. Software component agent checks the usage of CPU and MEMORY continually. To test the performance of MEMORY and CPU, RADAR fixes the threshold value of their usage. 


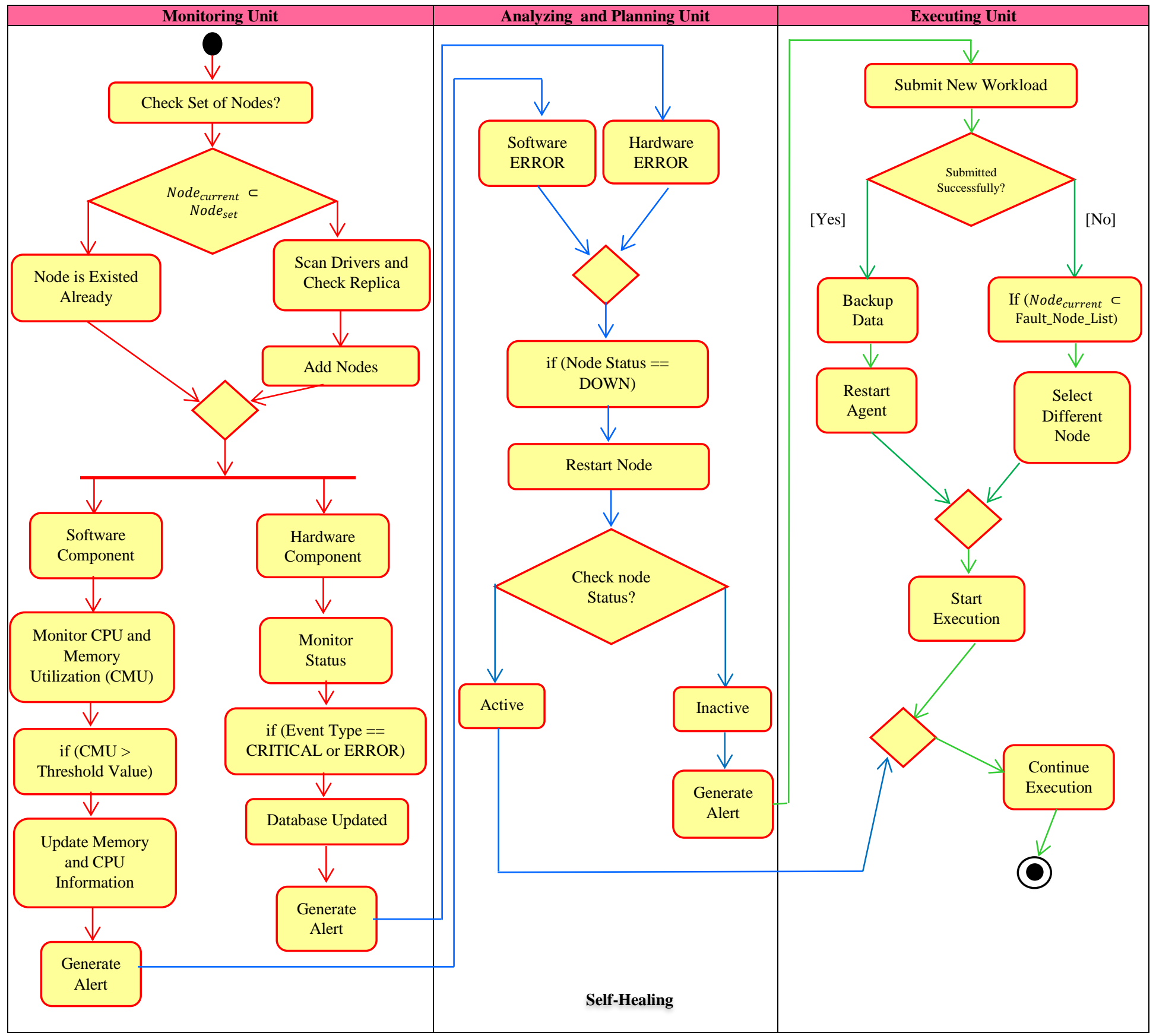

Figure 5: Process of Self-Healing

Figure 5 shows that the system generates an alert if the value CPU and MEMORY usage is greater than its threshold value. RADAR uses network agent to monitor the data transfer rate from one node to another. Manager nodes receive the status from all the processing nodes in a specific network. There can be network failure if processing nodes do not respond periodically.

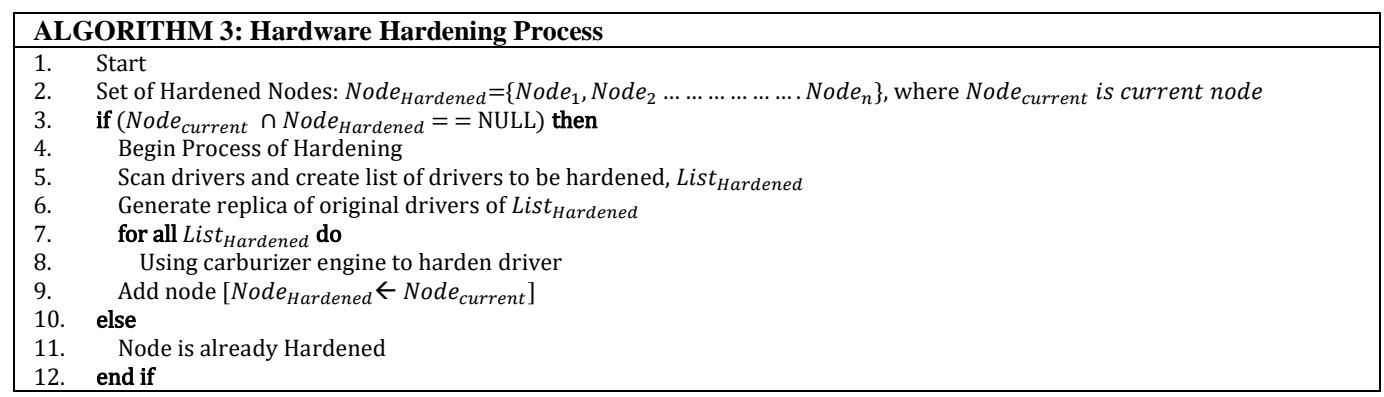

Analyzing and Planning unit analyses the alert for software or hardware, which is generated by software or hardware agent as shown in Figure 5. Hardware agent changes status of node $N$ as 'DOWN' if the event type is either 'ERROR' OR 'CRITICAL' and restarts the failed node using restart agent. Further, it measures the status of restarted node again and continues execution if the status of node changes to 'ACTIVE', otherwise generates alert and replaces the current node (down) with another stable node. Stability of a node is identified from their log information during their past performance. Software agent analyses an alert if the value of CPU and MEMORY usage is greater than its threshold value and restarts the node using restart agent as shown in Figure 5. Further, it 
measures the status of restarted node again and continues execution if the status of node changes to 'ACTIVE', otherwise generates alert for replacement of current node with a more stable node to continue execution. Network agent analyses the behavior of the network and finds out the reason of failure, which can be network breakage, physical damage, packet loss and lack of scalability in distributed networks. Then, network agent selects a plan from past network log to correct it. Restart agent reboots the system automatically to recover the system from hardware failures.

Execution module implements the plan by replacing the current node (down) with a different node, which is more stable among available nodes. This module also saves the state of the node and perform restarting if an error occurs during workload execution. Further, a faulty component will be replaced by new component if restart fails to recover from failure.

\section{Performance Evaluation}

Figure 6 shows the cloud testbed, which is used to evaluate the performance of RADAR. We have modeled and simulated a cloud environment using CloudSim toolkit [28]. For experimental setup, the computing nodes are simulated that resembles resource configuration as shown in Table 8 . Three servers with different configurations have been used to create virtual nodes in this experimental work. Each virtual node consists of Execution Components (ECs) for workload execution and cost for every EC is defined in C $\$ / E C$ time unit $(\mathrm{Sec})$. The configuration details of cloud testbed are shown in Table 8 and we have assigned manually access cost in Cloud Dollars (C\$). When EC have different capabilities then this cost does not essentially reflect the execution cost [29] [35] [37]. In this experimental work, access cost is translated into C\$ for each resource to find out the relative execution cost for workload execution.

Table 8: Configuration Details

\begin{tabular}{|l|c|c|c|c|c|}
\hline \multicolumn{1}{|c|}{ Resource Configuration } & Specifications & $\begin{array}{c}\text { Operating } \\
\text { System }\end{array}$ & $\begin{array}{c}\text { Number of Virtual } \\
\text { Node }\end{array}$ & $\begin{array}{c}\text { Number of } \\
\text { ECs }\end{array}$ & $\begin{array}{c}\text { Price (C\$/EC time } \\
\text { unit) }\end{array}$ \\
\hline Intel XEON E 52407-2.2 GHz & 2 GB RAM and 160 GB HDD & Linux & $2(1 \mathrm{~GB}$ and 60GB) & 6 & \\
\hline Intel Core i5-2310- $2.9 \mathrm{GHz}$ & $4 \mathrm{~GB}$ RAM and 160 GB HDD & Linux & $4(1 \mathrm{~GB}$ and 40 GB $)$ & 12 & 4 \\
\hline Intel Core 2 Duo - $2.4 \mathrm{GHz}$ & $6 \mathrm{~GB}$ RAM and 320 GB HDD & Windows & $6(1 \mathrm{~GB}$ and 50 GB $)$ & 18 & 3 \\
\hline
\end{tabular}

\subsection{Fault Management}

We have used Fault Injection Module (FIM-SIM) [30] to inject faults automatically to test the reliability of RADAR. FIM-SIM is working based on event-driven models and injects faults into the CloudSim [28] using different statistical distributions at runtime. We selected the Weibull Distribution [31] to inject faults in this research work. We have injected three types of faults: VM creation failures, host failures (Processing Elements failure and memory failure) and high-level failures like cloudlets failures (which are caused by any networking problem that CloudSim [28] cannot handle). The injection of faults affects cloud resources during the simulation period. Interested readers can find a detailed information about fault injection in [30]. Carburizer [32] is used in RADAR to harden the device drivers in Fault Manager to detect the faults and prevent the system from faults efficiently.

\subsection{Workloads}

For performance evaluation, we have selected four different types of cloud workload from every cluster of workloads as given in Table 3. Table 9 shows the different cloud workloads, which are considered to test the performance of RADAR. To find the experiment statistics, 3000 different workloads are executed. RADAR processes different workloads using the different number of resources to test its performance with different resource configuration. RADAR also maintains the details of every executed workload and stores into workload database, which can be used to test the efficiency of RADAR in future. Figure 6 shows the execution of "Performance Testing” workload, similarly, we executed other workloads [(i) Storage and Backup Data, (ii) Websites and (iii) Software Development and Testing] with the same experimental setup. Note: The detailed description of heterogeneous workloads is described in our previous research work [3].

Table 9: Details of Cloud Workloads

\begin{tabular}{|c|c|l|}
\hline Workload & \multicolumn{1}{|c|}{ Cluster } & \multicolumn{1}{c|}{ Description } \\
\hline Performance Testing & Compute (C1) & $\begin{array}{l}\text { RADAR processes and converts an image file (713 MB) to PNG format from JPG format } \\
\text { [33] [35]. The conversion of a single JPG file into PNG is considered as a workload (in } \\
\text { the form of Cloudlet). }\end{array}$ \\
\hline $\begin{array}{c}\text { Storage and Backup } \\
\text { Data }\end{array}$ & $\begin{array}{l}\text { Store larger amount of data (5 TB) and creates a backup of data [3] is considered as a } \\
\text { workload }\end{array}$ \\
\hline Websites & Communication (C3) & $\begin{array}{l}\text { A large number of users are accessing a website of Thapar Institute of Engineering and } \\
\text { Technology [http://www.thapar.edu] during Admission Period of the Year 2016 is } \\
\text { considered as a workload [3]. }\end{array}$ \\
\hline $\begin{array}{c}\text { Software Development } \\
\text { and Testing }\end{array}$ & Administration (C4) & $\begin{array}{l}\text { Developed and tested Agri-Info Software to find out the productivity of a crop [38] is } \\
\text { considered as a workload. }\end{array}$ \\
\hline
\end{tabular}




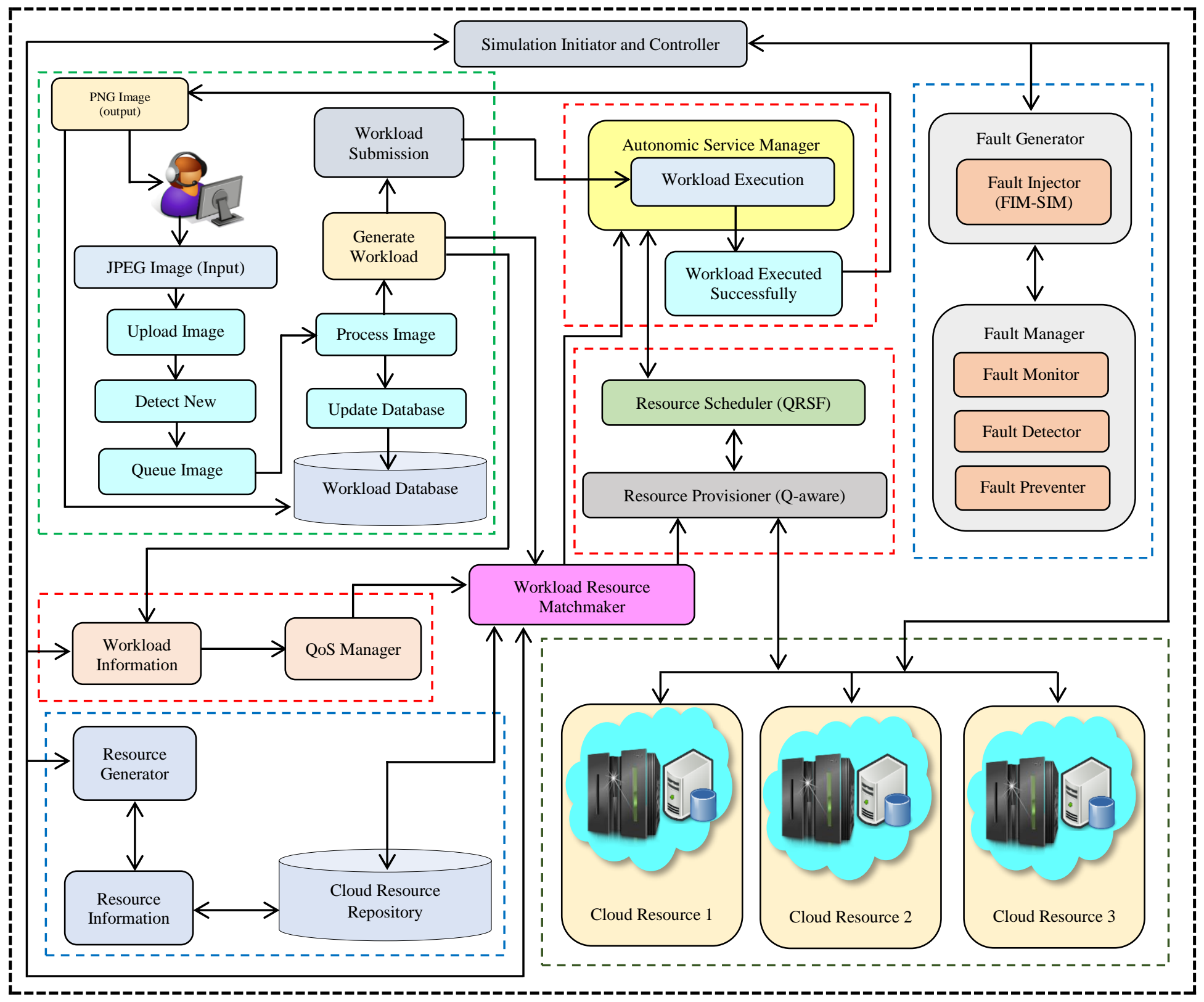

Figure 6: Cloud Testbed

\subsection{Experimental Results}

RADAR has been verified for two aspects: 1) self-configuring and 2) self-healing. We selected existing resource management techniques from literature to test the performance of RADAR in the cloud environment. All the experiments have been conducted with 500 to 3000 workloads to validate RADAR. We have considered 1500 workloads to measure the performance of QoS parameters with fault percentage for both self-configuring and self-healing. Fault percentage is defined as the percentage of faults existing in the system during execution of workloads and it is determined based on SLA violation rate. To test the capability of RADAR for detection of the failures, FIM-SIM [30] is used to inject different percentage of faults ( $0 \%$ to $5 \%)$. We provided standard error bars for every graph to show the variation in the experimental results.

\subsubsection{Self-configuring Verification}

We have selected two existing autonomic resource management techniques i.e. EASC [21] and CoTuner [25], to test the performance of RADAR in terms of resource contention, resource utilization, execution cost and SLA violation rate. Both EASC [21] and CoTuner [25] have been discussed and compared with RADAR in Section 2.

Test Case 1: Resource Contention - Figure 7 shows the variation of resource contention for RADAR, EASC and CoTuner with the increase in the number of workloads. As the number of workloads increases from 500 to 3000 workloads, the value of resource contention increases. The average value of resource contention in RADAR is $4.3 \%$ and $6.64 \%$ less than CoTuner and EASC respectively. We have considered the six different values of fault percentage to test the performance of RADAR. Figure 8 shows the variation of resource contention for RADAR, EASC and CoTuner with the different value of fault percentage ( $0 \%$ to $5 \%$ ). The average value of resource contention in RADAR is $7.79 \%$ and $9.11 \%$ less than EASC and CoTuner respectively. This is expected as the workload execution is done using RADAR, which is based on Q-aware [26]. Based on deadline and priority of workload, 
clustering of workloads is performed, and resources are provisioned for effective scheduling. This is also because of the low variation in execution time across various resources as the resource list that is obtained from the resource provisioning unit is already filtered using Q-aware [26].

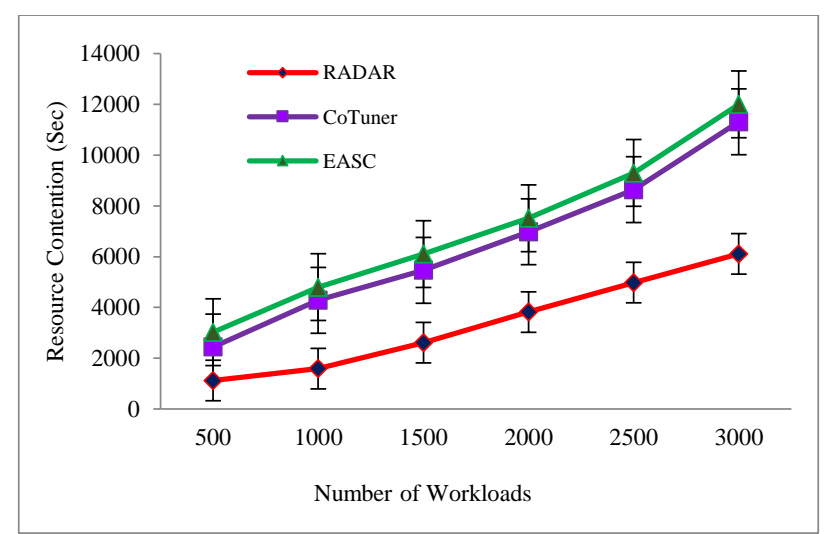

Figure 7: Resource Contention vs. Number of Workloads

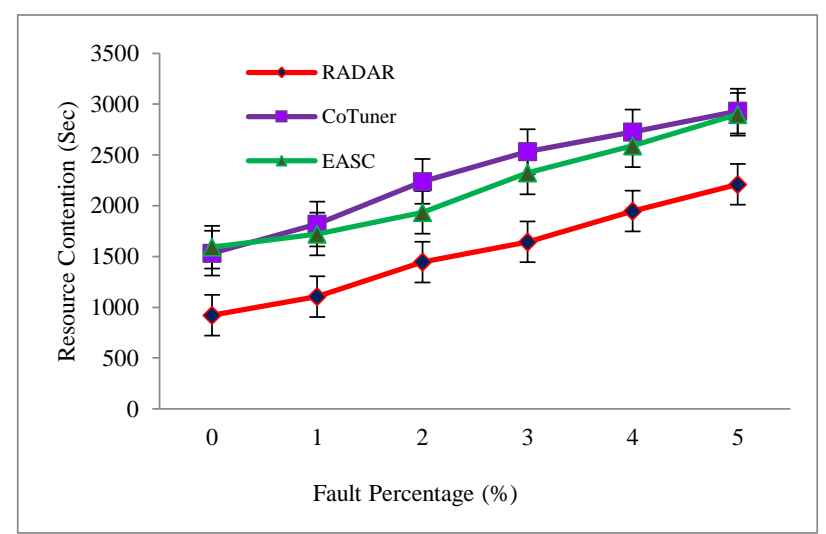

Figure 8: Resource Contention vs. Fault Percentage

Test Case 2: Resource Utilization - The value of Resource Utilization (RU) increases with the increase in the number of cloud workloads, as shown in Figure 9. The average value of RU in RADAR is $5.25 \%$ and $8.79 \%$ more than CoTuner and EASC respectively. Figure 10 shows the variation of RU with respect the fault percentage. For fault percentage, the average value of RU in RADAR is $4.16 \%$ and $6.93 \%$ more than CoTuner and EASC respectively. Based on QoS requirements of a specific workload, resource provisioning consumes little more time to find out the best resources [35], but later it increases the overall performance of RADAR. Therefore, underutilization and overutilization of resources will be assuaged or avoided, which reduces the further queuing time.

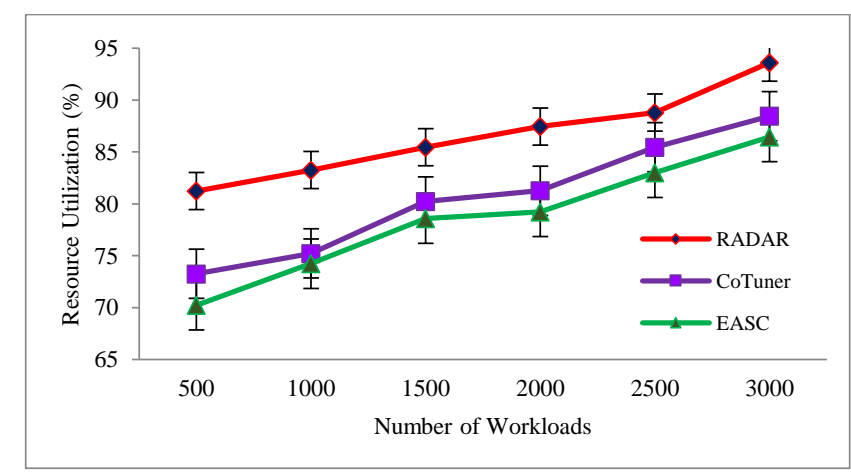

Figure 9: Resource Utilization vs. Number of Workloads

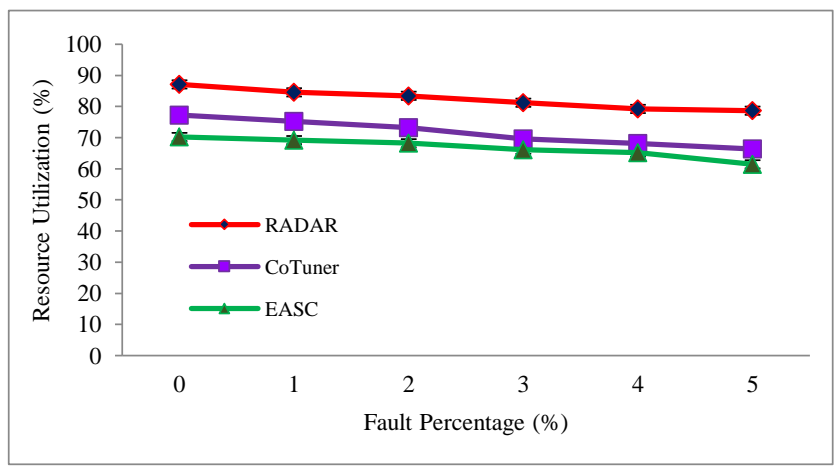

Figure 10: Resource Utilization vs. Fault Percentage 
Test Case 3: Execution Cost - The execution cost rises with the increase in the number of workloads, as shown in Figure 11. The average value of execution cost in RADAR is $3.33 \%$ and $5.83 \%$ less than CoTuner and EASC respectively. Figure 12 shows the variation of execution cost with the different values of fault percentage and the average value of execution cost in RADAR is $4.98 \%$ and $6.80 \%$ less than CoTuner and EASC respectively. The reason is that CoTuner and EASC do not consider the effect of other workloads in the resource scheduler at the time of workload submission but in RADAR, resource manager considers the effect of workloads in resource scheduler before execution of workload according to both user and resource provider's perspectives [26]. The other reason is that with the provisioned approach (Q-aware), due to a large number of workloads, these and later workloads had to be executed on left out resources, which may not be very cost effective.

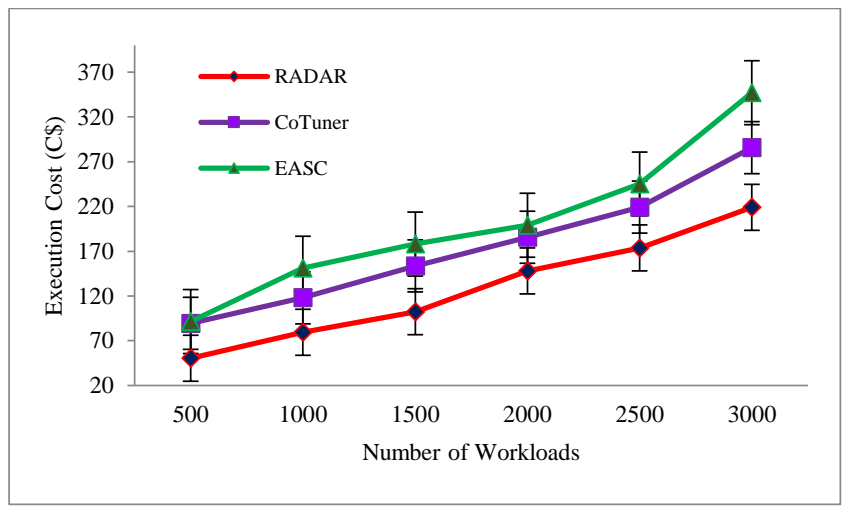

Figure 11: Execution Cost vs. Number of Workloads

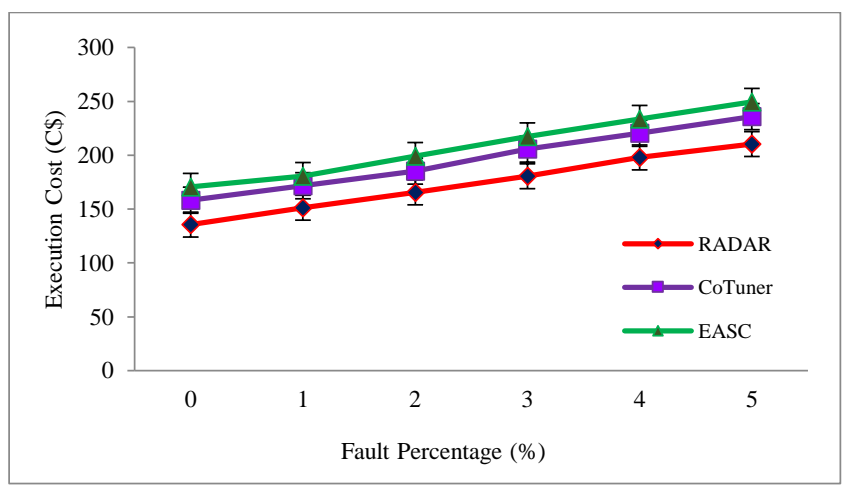

Figure 12: Execution Cost vs. Fault Percentage

Test Case 4: SLA Violation Rate - The impact of variation in the number of workloads on SLA Violation Rate (SVR) is analyzed. As shown in Figure 13, SVR increases with increase in the number of workloads and it shows that the average value of SVR in RADAR is $8.16 \%$ and $14.98 \%$ less than CoTuner and EASC respectively. Figure 14 shows the variation of SVR with different values of fault percentage and RADAR has 5.56\% and $6.16 \%$ less SVR than CoTuner and EASC respectively. This is because, RADAR uses admission control and reserve resources for execution of workloads in advance based on their QoS requirements specified in SLA document. Further, RADAR outperforms as it regulates the resources at runtime based on user's new QoS requirements during its execution to avoid SLA violation.

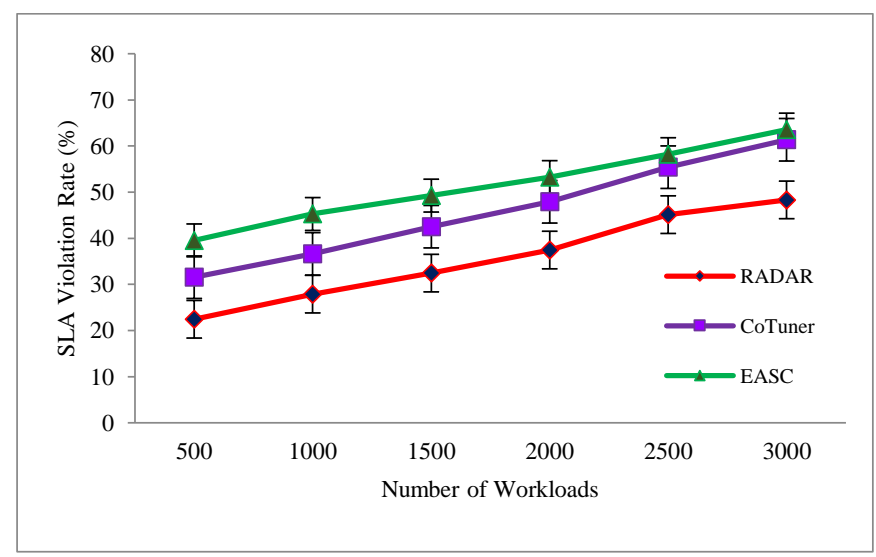

Figure 13: SLA Violation Rate vs. Number of Workloads 


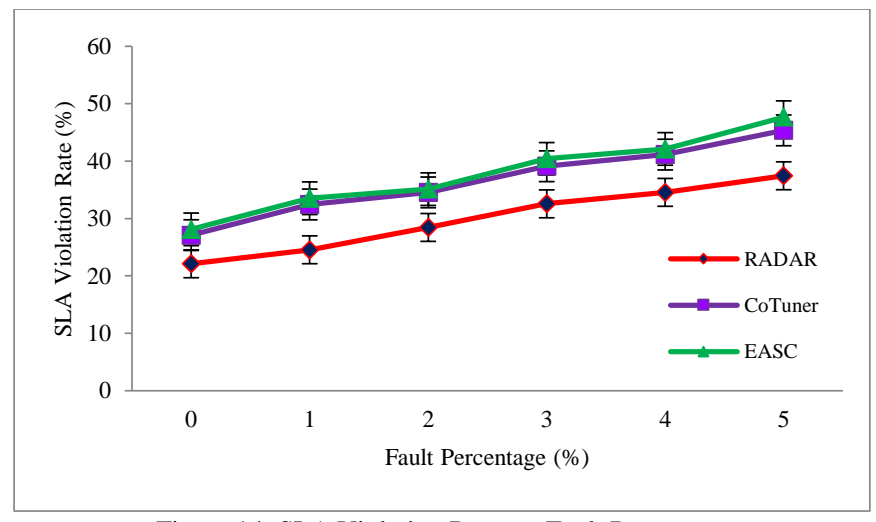

\subsubsection{Self-healing Verification}

Figure 14: SLA Violation Rate vs. Fault Percentage

We have selected two existing autonomic resource management techniques i.e. SH-SLA [5] and MASA [14], to test RADAR's performance in terms of reliability, fault detection rate, throughput, waiting time, execution time, energy consumption, availability and turnaround time. Both SH-SLA [5] and MASA [14] have been discussed and compared with RADAR in Section 2.

Test Case 1: Fault Detection Rate - Figure 15 shows the variation of Fault Detection Rate (FDR) with the different number of workloads. With an increase in the number of workloads, the value of FDR decreases. From 500 to 1500 cloud workloads, the value of FDR reduces, but RADAR performs better than SH-SLA and MASA. The average value of FDR in RADAR is $13.72 \%$ and $16.88 \%$ more than SH-SLA and MASA respectively. RADAR uses hardware hardening process to decrease the frequency of fault occurrence and it hardens the device drivers by using the concept of Carburizer [32], which keeps system's working without degradation of performance even though faults occur [39]. Once the hardening process is over, the status of nodes is forwarded to the monitor component (autonomic service manager) to prevent future faults. To avoid the same kind of future faults, RADAR replaces the hardened driver with an original driver if an alert is generated because of driver's misbehavior.

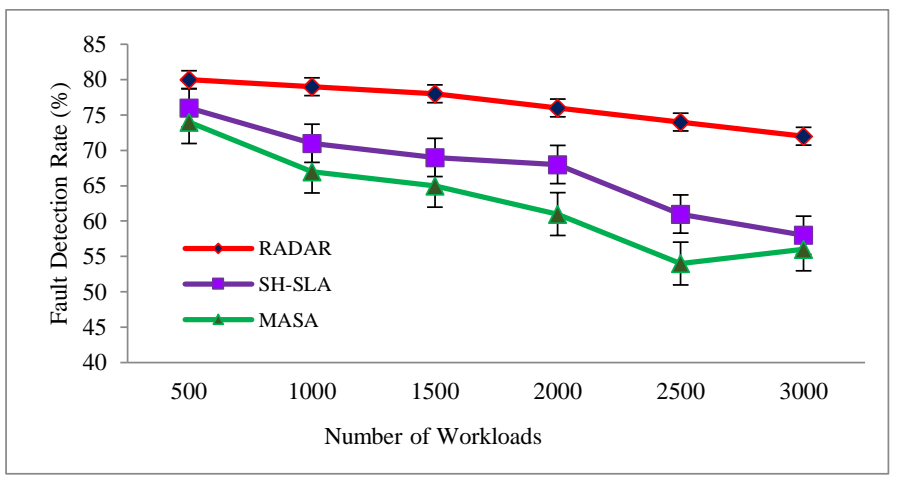

Figure 15: Fault Detection Rate Vs. Number of Workloads

Test Case 2: Throughput - Figure 16 shows the variation of throughput with the different number of workloads. The average value of throughput in RADAR is $11.1 \%$ and $14.50 \%$ more than SH-SLA and MASA respectively. We have injected a number of faults (fault percentage) to verify the throughput of RADAR with 1500 workloads. Figure 17 shows the comparison of throughput of RADAR with SH-SLA and MASA. From experimental result, it has been found that the maximum throughput at $0 \%$ fault percentage and minimum at 5\%. The average value of throughput in RADAR is $8.33 \%$ and $10.51 \%$ more throughput than SH-SLA and MASA respectively. RADAR identifies the software, hardware and network faults automatically and it also prevents the system from future faults, which improves the throughput of RADAR as compared to SH-SLA and MASA.

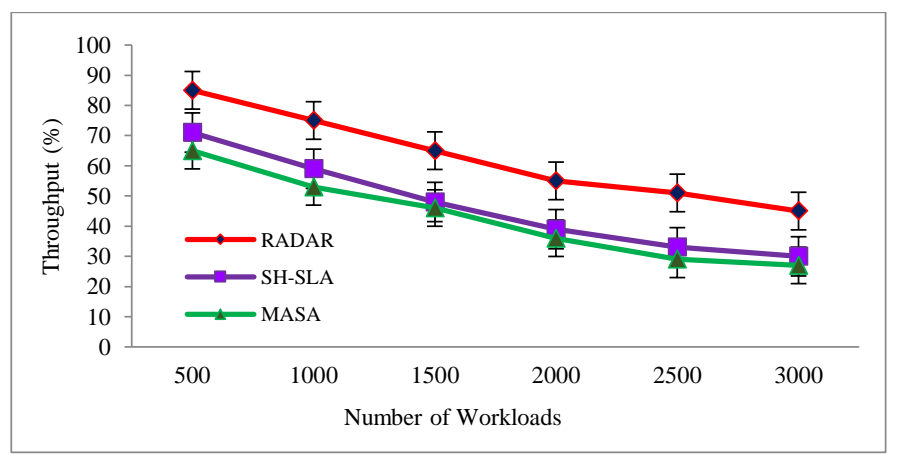

Figure 16: Throughput Vs. Number of Workloads 


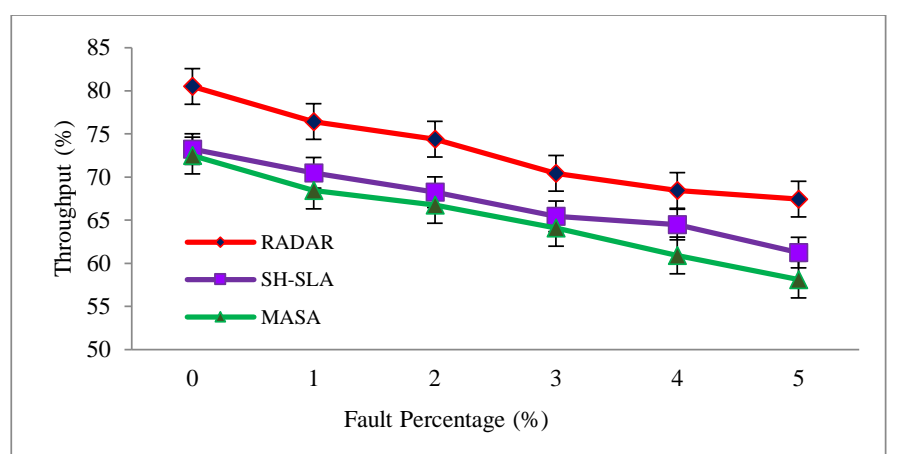

Figure 17: Throughput Vs. Fault Percentage

Test Case 3: Reliability - The value of reliability decreases with variation in the number of workloads, but RADAR performs better than SH-SLA and MASA as shown in Figure 18. The maximum reliability at 500 workloads is $91.45 \%$. The value of reliability in RADAR is $8.32 \%$ and $11.23 \%$ more than SH-SLA and MASA respectively. Figure 19 shows the variation of reliability with a different value of fault percentage and the value of reliability in RADAR is $13.26 \%$ and $14.31 \%$ more than SH-SLA and MASA respectively. The main difference between these works (SH-SLA and MASA) and ours (RADAR) is that none of them considers autonomic fault prevention mechanism for self-healing with dynamic checkpoint intervals, which uses RADAR. SH-SLA and MASA techniques focus on monitoring faults to maximize fault detection rate, whereas RADAR focuses on fault detection as well as prevention mechanisms. The efficient management of faults in RADAR improves the reliability of cloud services.

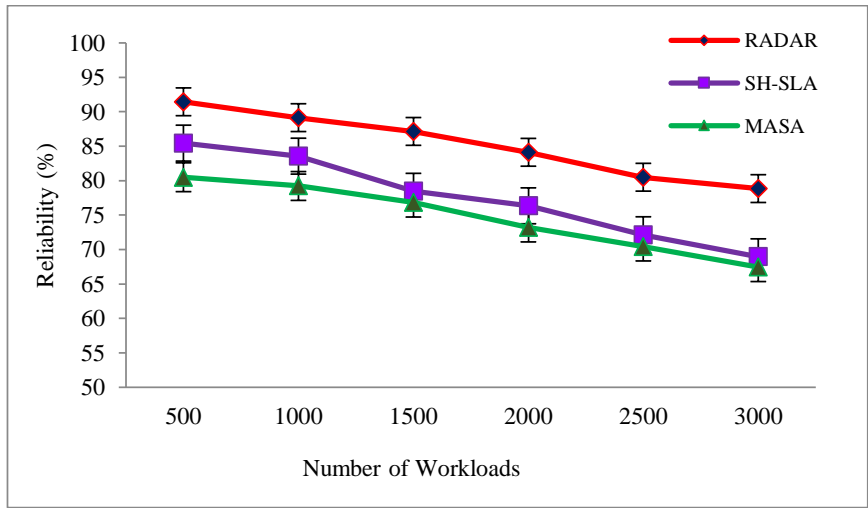

Figure 18: Reliability vs. Number of Workloads

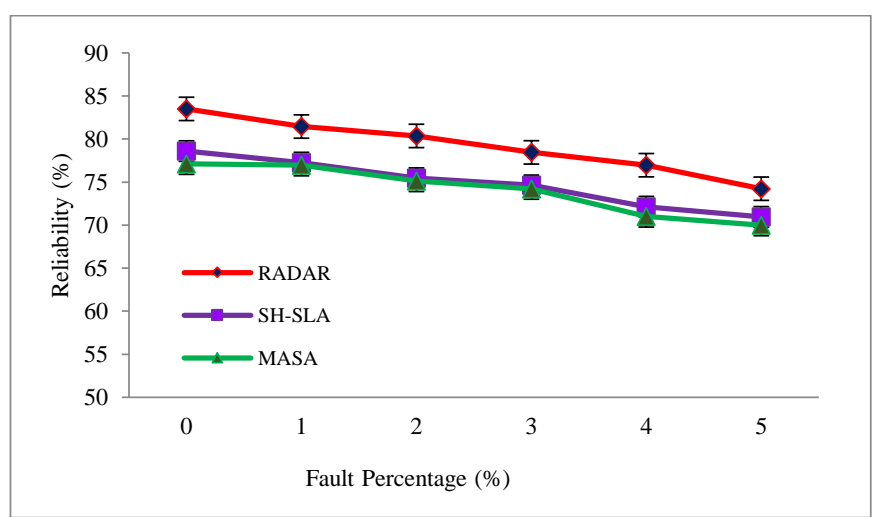

Figure 19: Reliability vs. Fault Percentage

Test Case 4: Availability - The value of availability for RADAR, SH-SLA and MASA is calculated and the value of availability decreases with increase in the number of workloads, as shown in Figure 20. The average value of availability in RADAR is 5.23\% and 5.96\% more than SH-SLA and MASA respectively. Figure 21 shows the variation of availability with the different value of fault percentage and the value of availability in RADAR is $3.45 \%$ and $4.46 \%$ more than SH-SLA and MASA respectively. This is expected as the recovering faulty task manages the faults efficiently in RADAR, which further improves the availability of cloud services. 


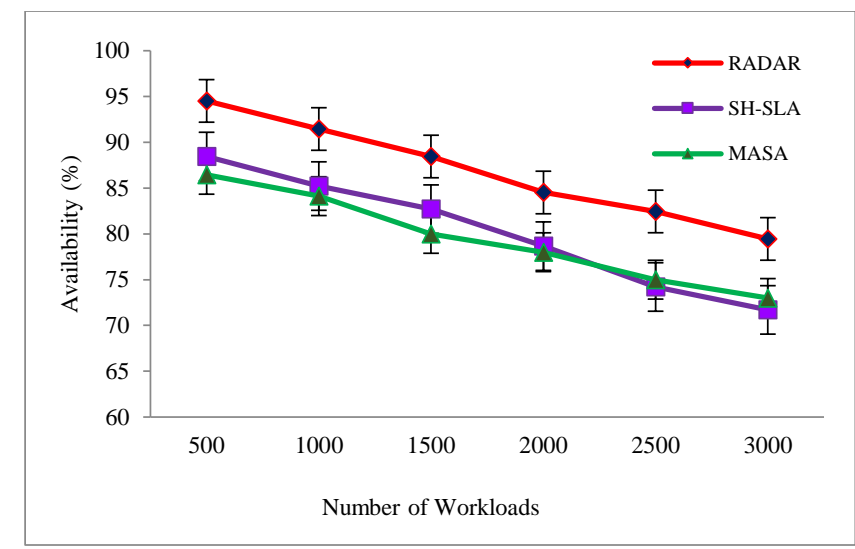

Figure 20: Availability vs. Number of Workloads

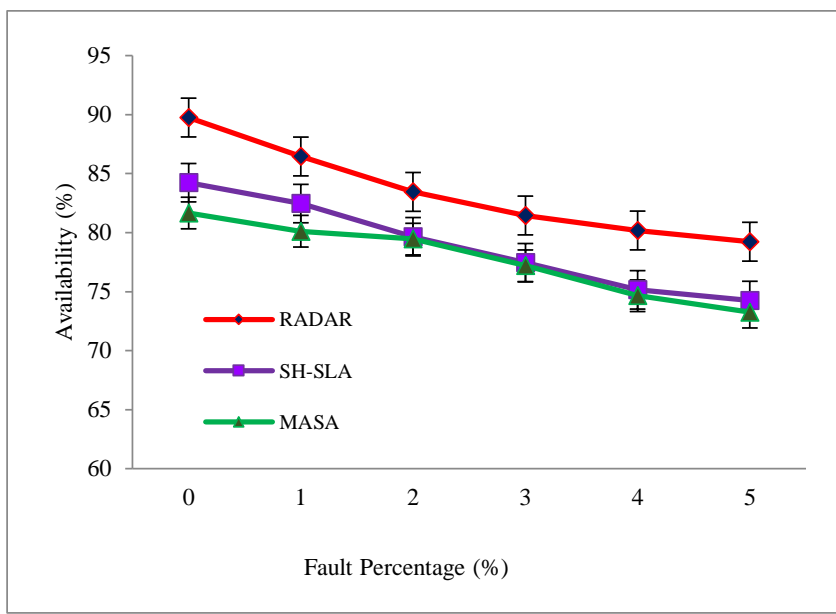

Figure 21: Availability vs. Fault Percentage

Test Case 5: Execution Time - As shown in Figure 22, the execution time increases with the increase in the number of workloads. The value of execution time in RADAR is $6.15 \%$ and $6.95 \%$ less than SH-SLA and MASA respectively. After 1500 workloads, execution time increases suddenly but RADAR produces better outcomes than SH-SLA and MASA. At 3000 workloads, the execution time in RADAR is $11.11 \%$ and $21.23 \%$ less than SH-SLA and MASA respectively. Figure 23 shows the variation of execution time with the different value of fault percentage and the value of execution time in RADAR is $10.23 \%$ and $19.98 \%$ less than SH-SLA and MASA respectively. This is expected as RADAR is keeping track of the state of all resources at each point of the time automatically which enables it to take an optimal decision (minimum execution time) than SH-SLA and MASA.

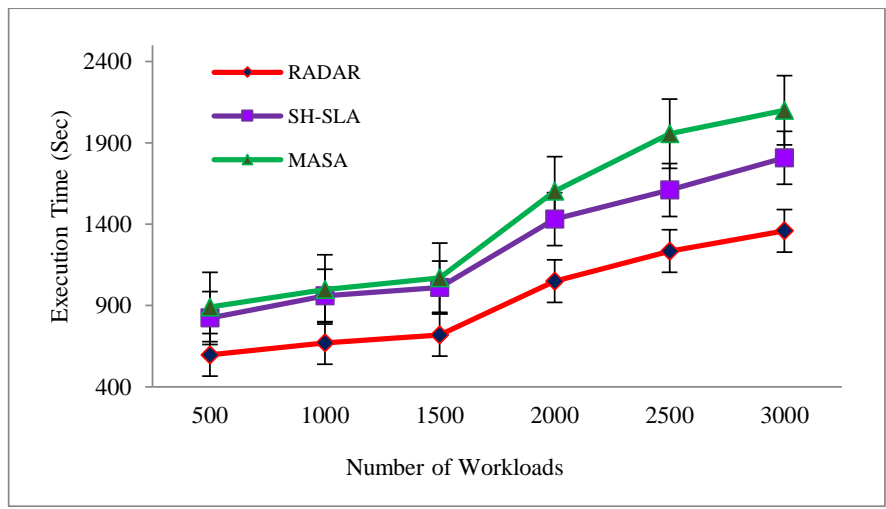

Figure 22: Execution Time vs. Number of Workloads 


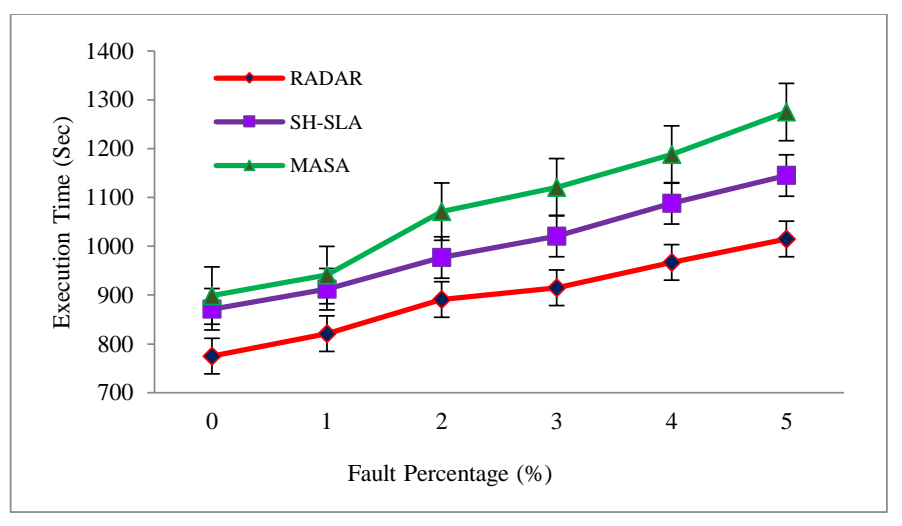

Figure 23: Execution Time vs. Fault Percentage

Test Case 6: Energy Consumption - With the different number of cloud workloads, energy consumption is calculated in kilo Watt hour (kWh) for RADAR, SH-SLA and MASA as shown in Figure 24. Energy consumption also increases, with the increasing number of cloud workloads. The minimum value of energy consumption is $46.12 \mathrm{kWh}$ at 500 workloads. The average value of energy consumption in RADAR is $8.11 \%$ and $9.73 \%$ less than SH-SLA and MASA respectively. Figure 25 shows the variation of energy consumption with the different value of fault percentage. Energy consumption in RADAR is $5.89 \%$ and $11.25 \%$ less than SH-SLA and MASA respectively. With the capability of automatically turning on and off nodes according to demands, RADAR provisions and schedules resources efficiently and intelligently for execution of clustered workloads instead of individual workloads. Further, workload clustering reduces the significant amount of network traffic which leads to reducing the number of active switches, which also reduces the wastage of energy.

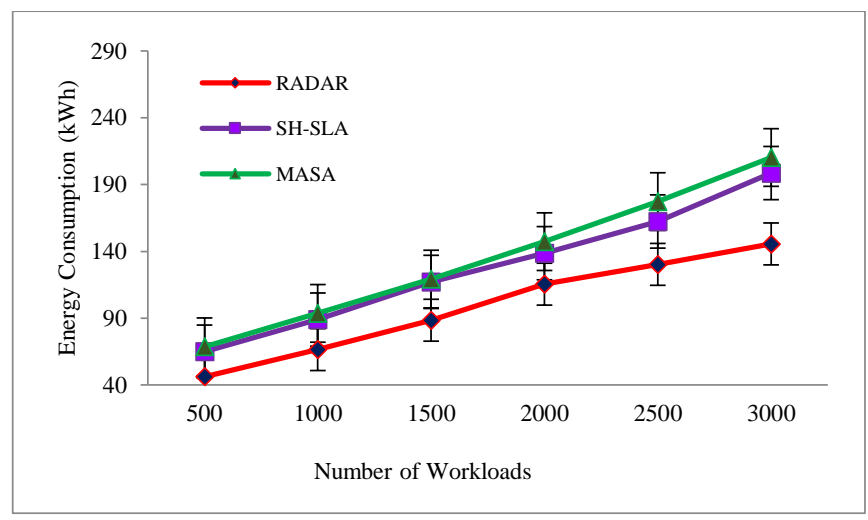

Figure 24: Energy Consumption vs. Number of Workloads

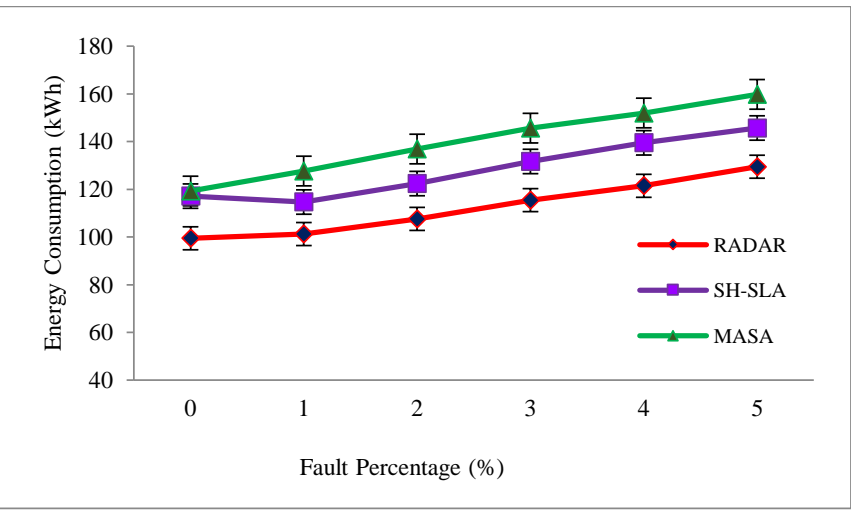

Figure 25: Energy Consumption vs. Fault Percentage

Test Case 7: Waiting Time - The performance of RADAR in terms of waiting time with the different number of workloads is verified. Figure 26 shows the variation of waiting time with the different number of workloads and the value of waiting time in RADAR is $15.22 \%$ and $19.75 \%$ less than SH-SLA and MASA respectively. The variation of waiting time with the different value of fault percentage is shown in Figure 27 and the value of waiting time in RADAR is $8.33 \%$ and 9.96\% less than SH-SLA and MASA respectively. The cause is that RADAR adjusts the provisioned resources dynamically according to the QoS requirements of workload to fulfill their required deadline, which also reduces the waiting time of workload in a queue. 


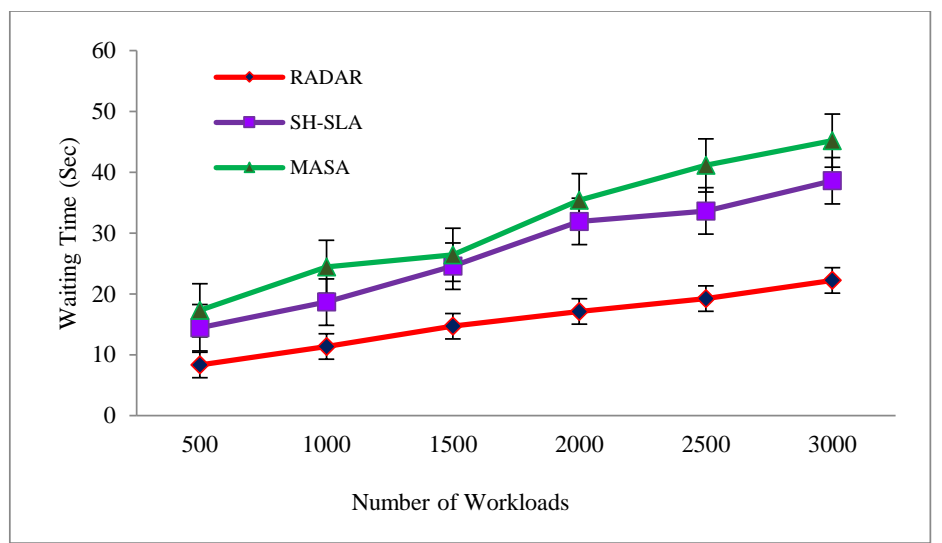

Figure 26: Waiting Time vs. Number of Workloads

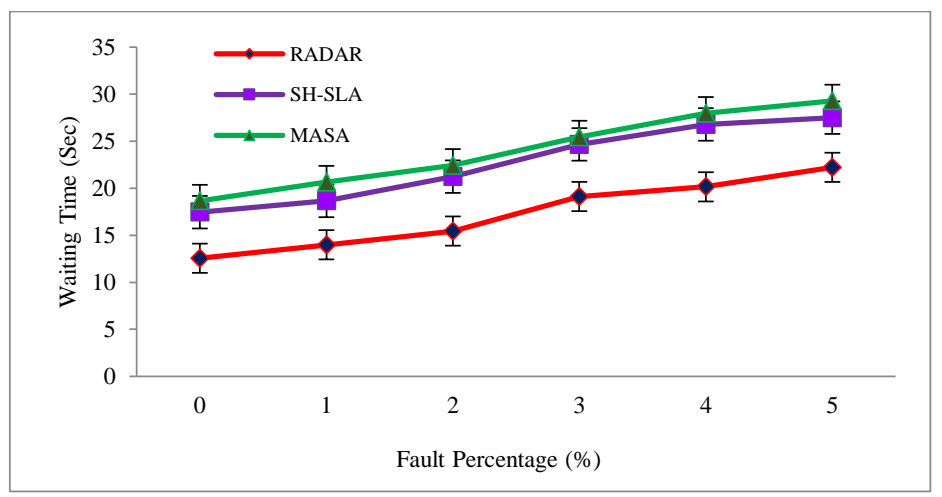

Figure 27: Waiting Time vs. Fault Percentage

Test Case 8: Turnaround Time - The value of turnaround time in RADAR with the different number of workloads is shown in Figure 28. The average value of turnaround time in RADAR is $13.33 \%$ and $17.45 \%$ less than SH-SLA and MASA respectively. Figure 29 shows the variation of turnaround time with the different value of fault percentage and the average value of turnaround time in RADAR is $7.14 \%$ and $12.75 \%$ less than SH-SLA and MASA respectively. SH-SLA and MASA consider scheduling of single queued workload, while RADAR considers clustering of workloads based on their QoS requirements. This is also because, RADAR is keeping track of the state of all resources at each point of the time automatically which enables it to take an optimal decision (minimum execution time) than SH-SLA and MASA.

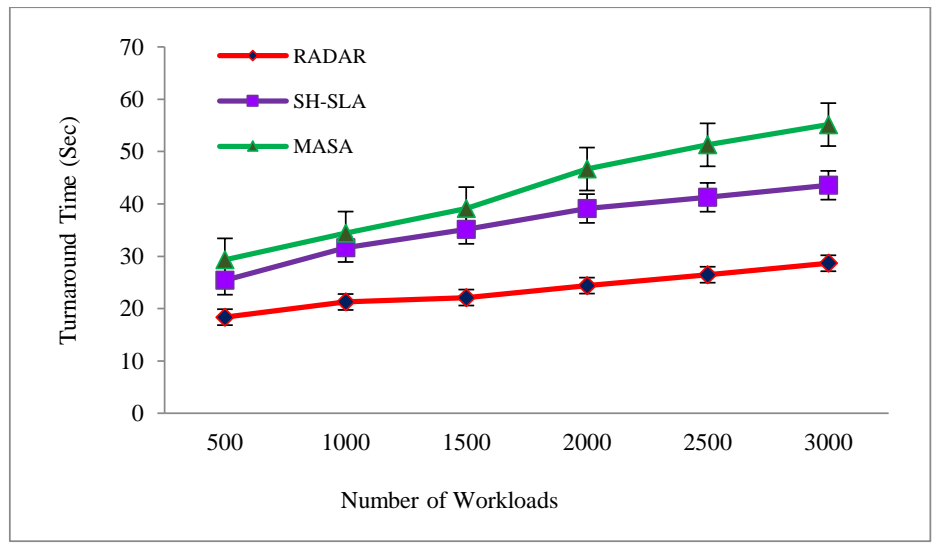

Figure 28: Turnaround Time vs. Number of Workloads 


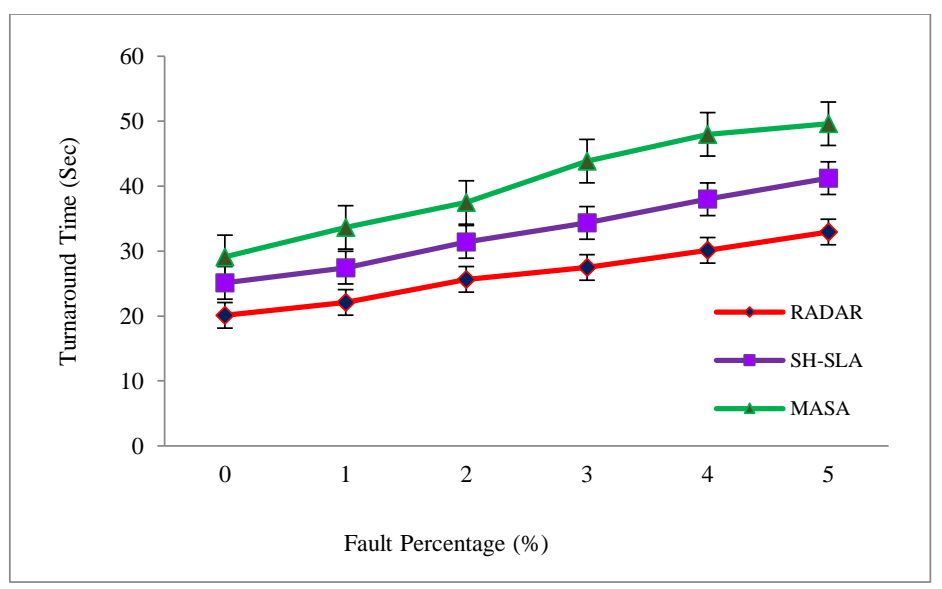

\subsection{Statistical Analysis}

Figure 29: Turnaround Time vs. Fault Percentage

Statistical significance of the results has been analyzed by Coefficient of Variation (CoV), which compares the different means and furthermore offers an overall analysis of the performance of RADAR used for creating the statistics. It states the deviation of the data as a proportion of its average value [29], and is calculated as follows [Eq. 33]:

$$
\mathrm{CoV}=\frac{S D}{\mathrm{M}} \times 100
$$

Where $S D$ is the Standard Deviation and $M$ is the Mean. CoV of fault detection rate of RADAR, SH-SLA and MASA is shown in Figure 30 . The range of $\mathrm{CoV}(0.48 \%-1.02 \%)$ for fault detection rate approves the stability of RADAR.

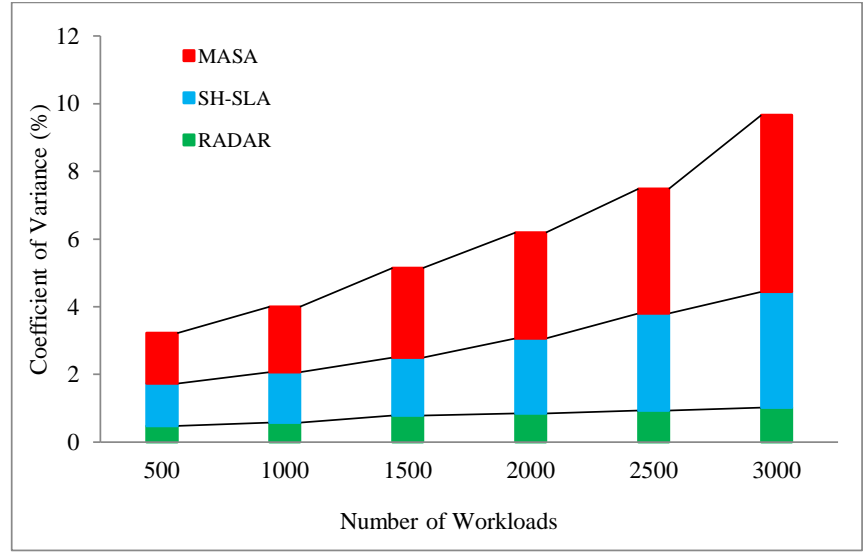

Figure 30: Coefficient of Variation for Fault Detection Rate

Value of CoV increases as the number of workloads increases. The small value of CoV signifies that RADAR is more efficient and stable in the management of cloud resources in those circumstances, where the quantity of workloads is varying. RADAR attained better results in cloud for fault detection rate and has been exhibited with respect to the number of workloads.

Table 10 describes the comparison of different QoS parameters, which are measured while the different number of resources/VMs are used to process various heterogeneous workloads (1500 and 3000). It shows that RADAR optimizes the QoS parameters with increase in the number of VMs gradually. RADAR executes 1500 workloads with one virtual node running on Server R1 and its execution completed in 422.2 seconds, while the same number of workloads finished in 342.6 seconds using 12 virtual nodes (2 virtual nodes running on R3, 4 virtual nodes running on R2 and 6 virtual nodes running on R1). Table 10 clearly shows that execution time decreases by adding additional virtual nodes. Similarly, by increasing the number of virtual nodes, other QoS parameters are also performing better.

\section{Conclusions and Future Work}

We have proposed an autonomic technique for cloud-based resources called RADAR to efficiently manage the provisioned cloud resources. RADAR improves the user satisfaction by maintaining SLA based on QoS requirements of cloud user and has an ability to manage resources automatically through properties of self-management, which are self-healing (find and react to sudden faults) and self-configuring (capability to readjust resources) with minimum human intervention. The performance of RADAR has been evaluated using CloudSim toolkit and experimental results show that RADAR performs better in terms of different QoS parameters and deals with software, network and hardware faults as compared to existing resource management techniques as shown in test cases. Experimental results demonstrate that RADAR improves the fault detection rate by $16.88 \%$, resource utilization by $8.79 \%$, throughput by $14.50 \%$, availability by $5.96 \%$ and reliability by $11.23 \%$ and it reduces the resource contention by $6.64 \%$, SLA violation rate by $14.98 \%$, execution time by $6.95 \%$, energy consumption by $9.73 \%$, waiting time by $19.75 \%$, turnaround time by $17.45 \%$ and execution cost by $5.83 \%$ as compared to resource management techniques. The small value of Coefficient of Variation 
$(\mathrm{CoV})$ signifies that RADAR is more efficient and stable in the management of cloud resources in those circumstances, where the quantity of workloads is varying. RADAR improves user satisfaction by fulfilling their QoS requirements and increases reliability and availability of cloud-based services. Further, RADAR can be extended to exhibit the other property of self-management such as self-protecting (detection and protection of cyber-attacks).

Table 10: Variation of QoS Parameters for Workload Distribution on different Number of Resources

\begin{tabular}{|c|c|c|c|c|c|c|c|c|c|c|c|c|c|c|c|c|}
\hline \multirow[t]{2}{*}{$\begin{array}{l}\text { Number of } \\
\text { Workloads }\end{array}$} & \multicolumn{3}{|c|}{$\begin{array}{c}\text { Number of } \\
\text { Resources }\end{array}$} & \multirow[t]{2}{*}{$\begin{array}{c}\text { Total } \\
\text { Virtual } \\
\text { Nodes } \\
\end{array}$} & \multirow[t]{2}{*}{$\begin{array}{c}\text { Execution } \\
\text { Time } \\
\text { (Sec) }\end{array}$} & \multirow[t]{2}{*}{$\begin{array}{l}\text { Execution } \\
\text { Cost }(C \$)\end{array}$} & \multirow[t]{2}{*}{$\begin{array}{c}\text { Waiting } \\
\text { Time } \\
\text { (Sec) }\end{array}$} & \multirow[t]{2}{*}{$\begin{array}{c}\text { Fault } \\
\text { Detection } \\
\text { Rate }(\%)\end{array}$} & \multirow[t]{2}{*}{$\begin{array}{c}\text { Throughput } \\
(\%)\end{array}$} & \multirow[t]{2}{*}{$\begin{array}{c}\text { Resource } \\
\text { Utilization } \\
(\%)\end{array}$} & \multirow[t]{2}{*}{$\begin{array}{c}\text { Energy } \\
\text { Consumption } \\
(\mathrm{kWh})\end{array}$} & \multirow[t]{2}{*}{$\begin{array}{l}\text { Resource } \\
\text { Contention } \\
\text { (Sec) }\end{array}$} & \multirow[t]{2}{*}{$\begin{array}{c}\text { SLA } \\
\text { Violation } \\
\text { Rate }(\%)\end{array}$} & \multirow[t]{2}{*}{$\begin{array}{l}\text { Turnaround } \\
\text { Time (Sec) }\end{array}$} & \multirow[t]{2}{*}{$\begin{array}{c}\text { Reliability } \\
(\%)\end{array}$} & \multirow[t]{2}{*}{$\begin{array}{c}\text { Availability } \\
(\%)\end{array}$} \\
\hline & R1 & R2 & R3 & & & & & & & & & & & & & \\
\hline 1500 & 1 & 0 & 0 & 1 & 422.2 & 65.71 & 8.72 & 85.23 & 82.22 & 78.26 & 18.56 & 2197 & 10.33 & 60.25 & 68.78 & 72.56 \\
\hline 1500 & 1 & 1 & 0 & 2 & 418.36 & 68.12 & 8.42 & 86.12 & 83.15 & 79.5 & 19.5 & 2143 & 10.03 & 61.65 & 70.05 & 74.45 \\
\hline 1500 & 2 & 1 & 0 & 3 & 412.65 & 70.12 & 7.91 & 86.88 & 84.66 & 81.62 & 22.61 & 2064 & 9.35 & 63.35 & 73.15 & 77.12 \\
\hline 1500 & 2 & 2 & 0 & 4 & 407.98 & 71.65 & 7.23 & 87.14 & 85.17 & 81.99 & 25.15 & 1927 & 9.02 & 63.93 & 77.14 & 81.62 \\
\hline 1500 & 3 & 2 & 0 & 5 & 401.15 & 72.99 & 6.69 & 87.59 & 86.22 & 82.45 & 26.99 & 1847 & 8.55 & 65.25 & 79.98 & 83.45 \\
\hline 1500 & 4 & 2 & 0 & 6 & 393.93 & 73.45 & 6.01 & 88.01 & 87.59 & 83.26 & 28.45 & 1768 & 8.13 & 67.18 & 80.05 & 84.62 \\
\hline 1500 & 4 & 2 & 1 & 7 & 381.65 & 75.64 & 5.70 & 89.5 & 88.23 & 84.76 & 29.55 & 1601 & 7.94 & 69.22 & 81.25 & 85.96 \\
\hline 1500 & 4 & 3 & 1 & 8 & 375.15 & 76.25 & 4.91 & 89.99 & 88.92 & 85.65 & 30.15 & 1498 & 7.71 & 70.12 & 82.14 & 86.12 \\
\hline 1500 & 5 & 3 & 2 & 10 & 359.74 & 79.98 & 3.61 & 92.22 & 89.95 & 86.89 & 32.75 & 1241 & 7.23 & 71.99 & 86.91 & 87.77 \\
\hline 1500 & 5 & 4 & 2 & 11 & 354.23 & 80.93 & 2.99 & 93.49 & 90.56 & 87.87 & 34.15 & 1205 & 7.01 & 72.05 & 87.25 & 88.25 \\
\hline 1500 & 6 & 4 & 2 & 12 & 342.2 & 81.66 & 2.46 & 94.45 & 91.62 & 88.65 & 35.97 & 1120 & 6.91 & 72.17 & 88.71 & 90.12 \\
\hline 3000 & 1 & 0 & 0 & 1 & 811.23 & 92.66 & 18.11 & 83.25 & 81.31 & 81.12 & 45.61 & 4236 & 16.17 & 76.26 & 65.71 & 52.65 \\
\hline 3000 & 1 & 1 & 0 & 2 & 845.26 & 98.11 & 16.91 & 84.25 & 81.85 & 81.95 & 47.26 & 4012 & 15.25 & 76.59 & 67.18 & 56.45 \\
\hline 3000 & 2 & 1 & 0 & 3 & 899.75 & 103.98 & 16.01 & 85.41 & 82.16 & 82.45 & 53.26 & 3883 & 14.93 & 77.18 & 69.37 & 58.72 \\
\hline 3000 & 2 & 2 & 0 & 4 & 955.65 & 107.45 & 15.26 & 85.98 & 82.98 & 82.88 & 57.45 & 3571 & 14.21 & 78.01 & 70.85 & 63.35 \\
\hline 3000 & 3 & 2 & 0 & 5 & 988.45 & 116.78 & 14.93 & 86.56 & 83.56 & 83.61 & 58.61 & 3243 & 13.55 & 79.50 & 71.12 & 66.78 \\
\hline 3000 & 4 & 2 & 0 & 6 & 1020.20 & 222.12 & 14.20 & 87.45 & 84.45 & 84.77 & 62.45 & 2961 & 13.12 & 80.12 & 72.56 & 69.92 \\
\hline 3000 & 4 & 2 & 1 & 7 & 1060.38 & 227.93 & 13.59 & 88.78 & 85.69 & 85.12 & 66.98 & 2719 & 12.89 & 81.66 & 75.45 & 73.26 \\
\hline 3000 & 5 & 3 & 2 & 10 & 1225.65 & 242.79 & 9.11 & 91.06 & 87.21 & 86.75 & 76.95 & 2105 & 10.26 & 83.97 & 81.39 & 83.45 \\
\hline 3000 & 5 & 4 & 2 & 11 & 1301.85 & 245.45 & 7.52 & 92.45 & 88.01 & 87.22 & 78.78 & 2022 & 10.05 & 84.15 & 83.06 & 85.50 \\
\hline 3000 & 6 & 4 & 2 & 12 & 1445.65 & 147.65 & 6.78 & 93.56 & 89.78 & 87.98 & 80.42 & 1968 & 9.94 & 84.68 & 84.69 & 87.45 \\
\hline
\end{tabular}

\section{Acknowledgement}

One of the authors, Dr. Sukhpal Singh Gill [Postdoctoral Research Fellow], gratefully acknowledges Discovery Project of Australian Research Council (ARC) (grant no. DP160102414), The University of Melbourne, Australia, for awarding him the Fellowship to carry out this research work. We would like to thank all the anonymous reviewers for their valuable suggestions. We thank Professor Rao Kotagiri, Adel N. Toosi, Bowen Zhou, Manmeet Singh and Amanpreet Singh for their comments on improving the paper.

\section{References}

[1] Sukhpal Singh and Inderveer Chana, "QoS-aware Autonomic Resource Management in Cloud Computing: A Systematic Review", "ACM Computing Surveys", Volume 48, Issue 3, pp. 1-46, 2015.

[2] Sukhpal Singh, Inderveer Chana Rajkumar Buyya, "STAR: SLA-aware Autonomic Management of Cloud Resources," in IEEE Transactions on Cloud Computing, pp.1-14, DOI: https://doi.org/10.1109/TCC.2017.2648788

[3] Sukhpal Singh, Inderveer Chana, Maninder Singh, and Rajkumar Buyya. "SOCCER: Self-Optimization of Energy-efficient Cloud Resources." Cluster Computing 19, no. 4 (2016): 1787-1800.

[4] Gang Chen, Hai Jin, Deqing Zou, Bing Bing Zhou, Weizhong Qiang, and Gang Hu. "Shelp: Automatic self-healing for multiple application instances in a virtual machine environment." In Cluster Computing (CLUSTER), 2010 IEEE International Conference on, pp. 97-106. IEEE, 2010.

[5] Ahmad Mosallanejad, Rodziah Atan, Masrah Azmi Murad, and Rusli Abdullah. "A hierarchical self-healing SLA for cloud computing." International Journal of Digital Information and Wireless Communications (IJDIWC) 4, no. 1 (2014): 43-52.

[6] Amal Alhosban, Khayyam Hashmi, Zaki Malik, and Brahim Medjahed. "Self-healing framework for cloud-based services." In Computer Systems and Applications (AICCSA), 2013 ACS International Conference on, pp. 1-7. IEEE, 2013.

[7] Rafael Ferreira da Silva, Rafael, Tristan Glatard, and Frédéric Desprez. "Self-healing of operational workflow incidents on distributed computing infrastructures." In Proceedings of the 2012 12th IEEE/ACM International Symposium on Cluster, Cloud and Grid Computing (ccgrid 2012), pp. 318-325. IEEE Computer Society, 2012

[8] Tristan Glatard, Carole Lartizien, Bernard Gibaud, Rafael Ferreira Da Silva, Germain Forestier, Frederic Cervenansky, Martino Alessandrini et al. "A virtual imaging platform for multi-modality medical image simulation." IEEE Transactions on Medical Imaging 32, no. 1 (2013): 110-118.

[9] Wenrui Li, Pengcheng Zhang, and Zhongxue Yang. "A framework for self-healing service compositions in cloud computing environments." In Web Services (ICWS), 2012 IEEE 19th International Conference on, pp. 690-691. IEEE, 2012.

[10] Joao Paulo Magalhaes, and Luis Moura Silva. "A framework for self-healing and self-adaptation of cloud-hosted web-based applications." In Cloud Computing Technology and Science (CloudCom), 2013 IEEE 5th International Conference on, vol. 1, pp. 555-564. IEEE, 2013.

[11] Xin, Rui. "Self-healing cloud applications." In Software Testing, Verification and Validation (ICST), 2016 IEEE International Conference on, pp. 389-390. IEEE, 2016.

[12] Ahmad Mosallanejad, Rodziah Atan, Rusli Abdullah, Masrha Azmi Murad, and Taghi Javdani. "HS-SLA: A hierarchical self-healing SLA model for cloud computing." In The Second International Conference on Informatics Engineering \& Information Science (ICIEIS2013), pp. 1-11. The Society of Digital Information and Wireless Communication, 2013.

[13] Erkuden Rios, Eider Iturbe, and Maria Carmen Palacios. "Self-healing Multi-Cloud Application Modelling." In Proceedings of the 12th International Conference on Availability, Reliability and Security, p. 93. ACM, 2017.

[14] Meriem Azaiez and Walid Chainbi. "A Multi-agent System Architecture for Self-Healing Cloud Infrastructure." In Proceedings of the International Conference on Internet of things and Cloud Computing, p. 7. ACM, 2016

[15] Alirio Santos de Sa, and Raimundo José de Araújo Macêdo. "QoS Self-configuring Failure Detectors for Distributed Systems." In DAIS, pp. 126-140. 2010.

[16] Michael Maurer, Ivona Brandic, and Rizos Sakellariou. "Adaptive resource configuration for Cloud infrastructure management." Future Generation Computer Systems 29, no. 2 (2013): 472-487.

[17] Gwen Salaün, Xavier Etchevers, Noel De Palma, Fabienne Boyer, and Thierry Coupaye. "Verification of a Self-configuration Protocol for Distributed Applications in the Cloud." In Assurances for Self-Adaptive Systems, pp. 60-79. Springer Berlin Heidelberg, 2013.

[18] Xavier Etchevers, Thierry Coupaye, Fabienne Boyer, and Noel De Palma. "Self-configuration of distributed applications in the cloud." In Cloud Computing (CLOUD), 2011 IEEE International Conference on, pp. 668-675. IEEE, 2011. 
[19] Silviu Panica, Marian Neagul, Ciprian Craciun, and Dana Petcu. "Serving legacy distributed applications by a self-configuring cloud processing platform." In Intelligent Data Acquisition and Advanced Computing Systems (IDAACS), 2011 IEEE 6th International Conference on, vol. 1, pp. 139-144. IEEE, 2011.

[20] Luca Sabatucci, Salvatore Lopes, and Massimo Cossentino. "A goal-oriented approach for self-configuring mashup of cloud applications." In Cloud and Autonomic Computing (ICCAC), 2016 International Conference on, pp. 84-94. IEEE, 2016.

[21] David Isaac Wolinsky, Yonggang Liu, Pierre St Juste, Girish Venkatasubramanian, and Renato Figueiredo. "On the design of scalable, self-configuring virtual networks." In Proceedings of the Conference on High Performance Computing Networking, Storage and Analysis, p. 13. ACM, 2009.

[22] Palden Lama and Xiaobo Zhou. "Aroma: Automated resource allocation and configuration of mapreduce environment in the cloud." In Proceedings of the 9th international conference on Autonomic computing, pp. 63-72. ACM, 2012.

[23] Ioannis Konstantinou, Verena Kantere, Dimitrios Tsoumakos, and Nectarios Koziris. "COCCUS: self-configured cost-based query services in the cloud." In Proceedings of the 2013 ACM SIGMOD International Conference on Management of Data, pp. 1041-1044. ACM, 2013.

[24] Luca Sabatucci, Salvatore Lopes, and Massimo Cossentino. "Self-configuring cloud application mashup with goals and capabilities." Cluster Computing (2017): 1-17.

[25] Xiangping Bu, Jia Rao, and Cheng-Zhong Xu. "Coordinated self-configuration of virtual machines and appliances using a model-free learning approach." IEEE transactions on parallel and distributed systems 24, no. 4 (2013): 681-690.

[26] Sukhpal Singh, and Inderveer Chana, "Q-aware: Quality of service based cloud resource provisioning", Computers \& Electrical Engineering, (47) (2015): 138-160. DOI: http://dx.doi.org/10.1016/j.compeleceng.2015.02.003

[27] Sukhpal Singh, and Inderveer Chana. QRSF: QoS-aware resource scheduling framework in cloud computing. The Journal of Supercomputing, 71(1) (2015), 241-292.

[28] Rodrigo N. Calheiros, Rajiv Ranjan, Anton Beloglazov, César AF De Rose and Rajkumar Buyya. "CloudSim: a toolkit for modeling and simulation of cloud computing environments and evaluation of resource provisioning algorithms." Software: Practice and experience 41, no. 1: 23-50, 2011.

[29] Sukhpal Singh Gill, Inderveer Chana, Maninder Singh and Rajkumar Buyya, "CHOPPER: An Intelligent QoS-aware autonomic resource management approach for cloud computing." Cluster Computing (2017): 1-39. DOI: https://doi.org/10.1007/s10586-017-1040-z

[30] Nita, Mihaela-Catalina, Florin Pop, Mariana Mocanu, and Valentin Cristea. "FIM-SIM: Fault injection module for CloudSim based on statistical distributions." Journal of telecommunications and information technology 4 (2014): 14.

[31] Jiaxi Liu,, Zhibo Wu, Jin Wu, Jian Dong, Yao Zhao, and Dongxin Wen. "A Weibull distribution accrual failure detector for cloud computing." PloS one 12, no. 3 (2017): e0173666.

[32] Asim Kadav, Matthew J. Renzelmann and Michael M. Swift. "Tolerating hardware device failures in software." In Proceedings of the ACM SIGOPS 22nd symposium on Operating systems principles, pp. 59-72. ACM, 2009.

[33] Thieling, Lothar, Andre Schuer, Georg Hartung, and Gregor Buchel. "Embedded image processing system for cloud-based applications." In Systems, Signals and Image Processing (IWSSIP), 2014 International Conference on, pp. 163-166. IEEE, 2014.

[34] Tracy D. Braun, Howard Jay Siegel, Noah Beck, Ladislau L. Bölöni, Muthucumaru Maheswaran, Albert I. Reuther, James P. Robertson et al. "A comparison of eleven static heuristics for mapping a class of independent tasks onto heterogeneous distributed computing systems." Journal of Parallel and Distributed computing 61, no. 6 (2001): 810-837.

[35] Sukhpal Singh Gill, Rajkumar Buyya, Inderveer Chana, Maninder Singh, and Ajith Abraham. "BULLET: Particle Swarm Optimization Based Scheduling Technique for Provisioned Cloud Resources." Journal of Network and Systems Management: Volume 26, Issue 2, pp 361-400, 2018.

[36] Shoukat Ali, Howard Jay Siegel, Muthucumaru Maheswaran, Debra Hensgen and Sahra Ali. "Representing task and machine heterogeneities for heterogeneous computing systems." Tamkang J Sci Eng, 3, no. 3 (2000): 195-207.

[37] Gill, Sukhpal Singh, and Rajkumar Buyya. "Resource Provisioning Based Scheduling Framework for Execution of Heterogeneous and Clustered Workloads in Clouds: from Fundamental to Autonomic Offering." Journal of Grid Computing (2018): 1-33. DOI: https://doi.org/10.1007/s10723-017-9424-0

[38] Gill, Sukhpal Singh, Inderveer Chana, and Rajkumar Buyya. "IoT Based Agriculture as a Cloud and Big Data Service: The Beginning of Digital India." Journal of Organizational and End User Computing (JOEUC) 29, no. 4 (2017): 1-23.

[39] Inderpreet Chopra and Maninder Singh. 2014. SHAPE--an approach for self-healing and self-protection in complex distributed networks. J. Supercomput. 67, 2 (February 2014), 585-613 